CRYSTALLOGRAPHIC COMMUNICATIONS

ISSN 2056-9890

Received 8 May 2015

Accepted 17 May 2015

Edited by D.-J. Xu, Zhejiang University (Yuquan Campus), China

Keywords: crystal structure; binuclear copper(II) complex; oxamide complex; 1,10-phenanthroline-5,6-dione; hydrogen bonding; $\pi-\pi$ stacking

CCDC reference: 1401557

Supporting information: this article has supporting information at journals.iucr.org/e

\section{Crystal structure of aqua $\{\mu-\mathrm{N}$-[3-(dimethylamino)- propyl]- $N^{\prime}-2$-(oxidophenyl)oxamidato\}(1,10-phen- anthroline-5,6-dione)dicopper(II) perchlorate hemihydrate}

\author{
Xin Zhang, Yan-Tuan Li and Zhi-Yong Wu*
}

Key Laboratory of Marine Drugs, Ministry of Education of China, School of Medicine and Pharmacy, Ocean University of China, Qingdao 266003, People's Republic of China. *Correspondence e-mail: wuzy@ouc.edu.cn

The title compound, $\left[\mathrm{Cu}_{2}\left(\mathrm{C}_{13} \mathrm{H}_{16} \mathrm{~N}_{3} \mathrm{O}_{3}\right)\left(\mathrm{C}_{12} \mathrm{H}_{6} \mathrm{~N}_{2} \mathrm{O}_{2}\right)\left(\mathrm{H}_{2} \mathrm{O}\right)\right] \mathrm{ClO}_{4} \cdot 0.5 \mathrm{H}_{2} \mathrm{O}$, consists of a cis-oxamide-bridged binuclear $\mathrm{Cu}^{\mathrm{II}}$ complex cation, a perchlorate anion and half a solvent water molecule. One $\mathrm{Cu}^{\text {II }}$ cation is $N, N^{\prime}, N^{\prime \prime}, O$-chelated by an $N$-[3-(dimethylamino)propyl]- $N^{\prime}$-(2-hydroxyphenyl)oxamide trianion in a distorted square-planar geometry, whereas the other $\mathrm{Cu}^{\mathrm{II}}$ cation is $O, O^{\prime}$ chelated by the oxamide moiety of the anion and $N, N^{\prime}$-chelated by a 1,10 phenanthroline-5,6-dione molecule, and a water molecule further coordinates the second $\mathrm{Cu}^{\mathrm{II}}$ cation, completing a distorted square-pyramidal coordination geometry. In the crystal, classical $\mathrm{O}-\mathrm{H} \cdots \mathrm{O}$ hydrogen bonds, weak $\mathrm{C}-\mathrm{H} \cdots \mathrm{O}$ hydrogen-bonding interactions and $\pi-\pi$ stacking interactions link the complex cations, anions and solvent water molecules into a three-dimensional supramolecular architecture. In the crystal, the dimethylaminopropyl unit of the oxamide anion is disordered over two positions with an occupancy ratio of 0.561 (11):0.439 (11); the solvent water molecule is also disordered over two positions, the occupancy ratio being 0.207 (10):0.293 (10).

\section{Chemical context}

It is known that oxamide ligands could be good candidates for forming polynuclear complexes because of their versatile coordinating abilities (Ojima \& Nonoyama, 1988; Ruiz et al., 1999). Therefore, many oxamide complexes and their properties have been investigated extensively (Messori et al., 2003; Wang et al., 2013; Li et al., 2011).

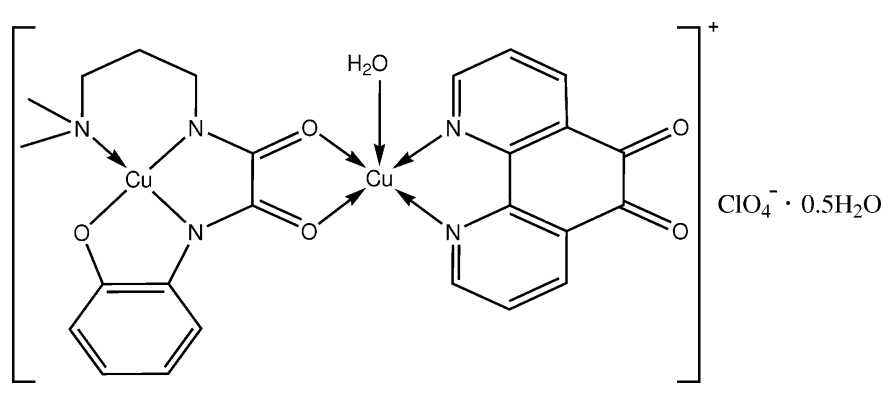

1,10-Phenanthroline-5,6-dione (Phdo) is a multifaceted ligand since the structure and electronic properties thereof incorporate the features of the diimine and quinone functionalities (Girgis et al., 1975; Calderazzo et al., 2002). Consequently, as part of our systematic study of asymmetrical bis-substituent oxamide complexes and the influence of structures on the DNA-binding properties thereof ( $\mathrm{Li}$ et al., 2012; Zhang et al., 2013), we selected $N$-[3-(dimethylamino)- 


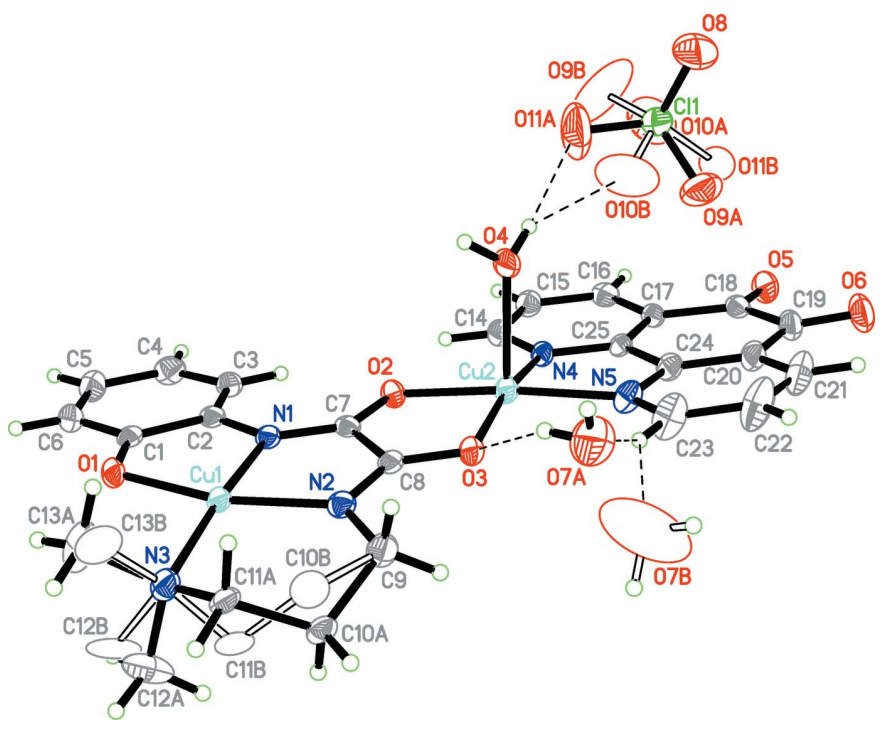

Figure 1

The molecular structure of the title compound with displacement ellipsoids drawn at the $50 \%$ probability level. For clarity, disordered atoms are represented in a different style and the $\mathrm{H}$ atoms on disordered carbon atoms have been omitted. Dashed lines indicate hydrogen bonds.

propyl]- $N^{\prime}$-2-(oxidophenyl)oxamide $\left(\mathrm{H}_{3}\right.$ Dmapox) as a bridging ligand and Phdo as a terminal ligand to synthesize the title binuclear copper(II) complex, $\left[\mathrm{Cu}_{2}(\text { Dmapox })(\mathrm{Phdo}) \mathrm{H}_{2} \mathrm{O}\right]^{+}$$\mathrm{ClO}_{4}{ }^{-} \cdot 0.5 \mathrm{H}_{2} \mathrm{O}$. Its crystal structure and supramolecular structure are reported here.

\section{Structural commentary}

The title compound consists of a binuclear $\mathrm{Cu}^{\mathrm{II}}$ complex cation, a perchlorate anion and half of a solvent water molecule (Fig. 1). Two copper(II) ions are bridged by a cisoxamido group. The $\mathrm{Cu} 1 \mathrm{atom}$, located at the inner site of the oxamide ligand, has a distorted square-planar geometry and is displaced from the coordination plane by 0.0454 (15) $\AA$, which is consistent with structures reported previously (Gao \& Wang, 2010; Lu et al., 2011). The two exo-oxygen atoms of the oxamide ligand and two nitrogen atoms of the Phdo molecule chelate the $\mathrm{Cu} 2$ atom, forming the basal coordination plane [the maximum deviation being 0.0384 (14) $\AA$ for N4], and a water molecule (O4) occupies the apical position, completing a distorted square-pyramidal coordination geometry with a $\tau$ value of 0.06 (Addison et al., 1984). The $\mathrm{Cu}-\mathrm{O}$ distance of 2.213 (3) $\AA$ in the apical direction is longer than those in the basal plane by 0.261 (4) and 0.266 (4) $\AA$ (Table 1). The $\mathrm{Cu} 2$ atom is displaced by $0.1610(15) \AA$ from the basal plane towards the apex.

The hexadentate oxamide anion, Dmapox ${ }^{3-}$, bridges the two copper(II) cations with three planar five-membered chelate rings and one six-membered ring, the latter being disordered over two positions. The puckering parameters of the first component (containing atoms $\mathrm{C} 10 \mathrm{~A}$ and $\mathrm{C} 11 \mathrm{~A}$ ) are $Q=0.554(8) \AA, \theta=47.6(6)^{\circ}$ and $\varphi=206.0(7)^{\circ}$, and those of
Table 1

Selected bond lengths $(\AA)$.

\begin{tabular}{llll}
\hline $\mathrm{Cu} 1-\mathrm{O} 1$ & $1.950(3)$ & $\mathrm{Cu} 2-\mathrm{O} 3$ & $1.947(3)$ \\
$\mathrm{Cu} 1-\mathrm{N} 1$ & $1.932(3)$ & $\mathrm{Cu} 2-\mathrm{O} 4$ & $2.213(3)$ \\
$\mathrm{Cu} 1-\mathrm{N} 2$ & $1.982(3)$ & $\mathrm{Cu} 2-\mathrm{N} 4$ & $1.989(3)$ \\
$\mathrm{Cu} 1-\mathrm{N} 3$ & $2.013(3)$ & $\mathrm{Cu} 2-\mathrm{N} 5$ & $1.991(3)$ \\
$\mathrm{Cu} 2-\mathrm{O} 2$ & $1.952(3)$ & & \\
\hline
\end{tabular}

Table 2

Hydrogen-bond geometry $\left(\AA{ }^{\circ}\right)$.

\begin{tabular}{lllll}
\hline$D-\mathrm{H} \cdots A$ & $D-\mathrm{H}$ & $\mathrm{H} \cdots A$ & $D \cdots A$ & $D-\mathrm{H} \cdots A$ \\
\hline $\mathrm{O} 4-\mathrm{H} 4 A \cdots \mathrm{O} 1^{\mathrm{i}}$ & 0.84 & 1.89 & $2.666(3)$ & 153 \\
$\mathrm{O} 4-\mathrm{H} 4 B \cdots \mathrm{O} 10 B$ & 0.91 & 1.93 & $2.740(8)$ & 146 \\
$\mathrm{O} 4-\mathrm{H} 4 B \cdots \mathrm{O} 11 A$ & 0.91 & 2.10 & $3.009(7)$ & 171 \\
$\mathrm{O} 7 A-\mathrm{H} 7 A \cdots \mathrm{O} 3$ & 0.86 & 2.51 & $3.27(2)$ & 147 \\
$\mathrm{O} 7 B-\mathrm{H} 7 D \cdots 5^{\mathrm{ii}}$ & 0.86 & 2.51 & $3.305(18)$ & 153 \\
$\mathrm{C} 3-\mathrm{H} 3 \cdots 8^{\mathrm{iii}}$ & 0.93 & 2.52 & $3.193(5)$ & 130 \\
$\mathrm{C} 15-\mathrm{H} 15 \cdots \mathrm{O} 9 A^{\mathrm{iii}}$ & 0.93 & 2.55 & $3.335(7)$ & 142 \\
$\mathrm{C} 15-\mathrm{H} 15 \cdots \mathrm{O} 11 B^{\mathrm{iii}}$ & 0.93 & 2.49 & $3.384(10)$ & 162 \\
$\mathrm{C} 16-\mathrm{H} 16 \cdots 6^{\mathrm{iii}}$ & 0.93 & 2.54 & $3.195(5)$ & 128 \\
$\mathrm{C} 10 A-\mathrm{H} 10 A \cdots \mathrm{O} 6^{\mathrm{ii}}$ & 0.97 & 2.44 & $3.202(9)$ & 135 \\
$\mathrm{C} 11 A-\mathrm{H} 11 A \cdots \mathrm{O} 4^{\text {iv }}$ & 0.97 & 2.54 & $3.409(7)$ & 150 \\
$\mathrm{C} 13 A-\mathrm{H} 13 B \cdots \mathrm{O} 4^{\mathrm{i}}$ & 0.96 & 2.49 & $3.382(16)$ & 154 \\
$\mathrm{C} 21-\mathrm{H} 21 \cdots \mathrm{O} 10 A^{\mathrm{v}}$ & 0.93 & 2.53 & $3.277(8)$ & 138 \\
$\mathrm{C} 23-\mathrm{H} 23 \cdots \mathrm{O} 7 A$ & 0.93 & 2.30 & $3.154(19)$ & 152 \\
$\mathrm{C} 23-\mathrm{H} 23 \cdots \mathrm{O} 7 B$ & 0.93 & 2.49 & $3.31(2)$ & 147 \\
\hline
\end{tabular}

Symmetry codes: (i) $-x+1,-y+1,-z$; (ii) $-x, y+\frac{1}{2},-z+\frac{1}{2}$; (iii) $x,-y+\frac{1}{2}, z-\frac{1}{2}$; (iv) $-x+1, y+\frac{1}{2},-z+\frac{1}{2} ;$ (v) $x,-y+\frac{1}{2}, z+\frac{1}{2}$.

the other are $Q=0.565(11) \AA, \theta=123.4(8)^{\circ}$ and $\varphi=38.8(9)^{\circ}$; both suggest an approximate half-chair conformation.

\section{Supramolecular features}

Besides classical $\mathrm{O}-\mathrm{H} \cdots \mathrm{O}$ hydrogen bonds, weak $\mathrm{C}-\mathrm{H} \cdots \mathrm{O}$ hydrogen bonds and aromatic stacking interactions are important to the supramolecular structure. As illustrated in Fig. 2, two symmetry-related binuclear cations link each other, forming a dimer by hydrogen bonds between the coordinating water molecules and phenolic oxygen atoms (Table 2). Then

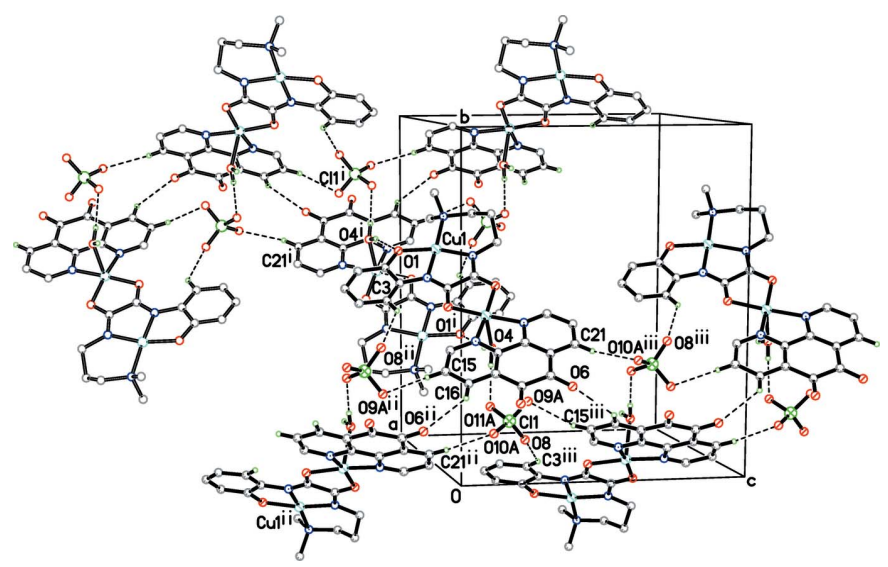

Figure 2

The two-dimensional wave-like hydrogen-bonding network constructed by classical $\mathrm{O}-\mathrm{H} \cdots \mathrm{O}$ and weak $\mathrm{C}-\mathrm{H} \cdots \mathrm{O}$ interactions [symmetry codes: (i) $1-x, 1-y,-z$; (ii) $x, \frac{1}{2}-y, z-\frac{1}{2}$; (iii) $\left.x, \frac{1}{2}-y, z+\frac{1}{2}\right]$. 


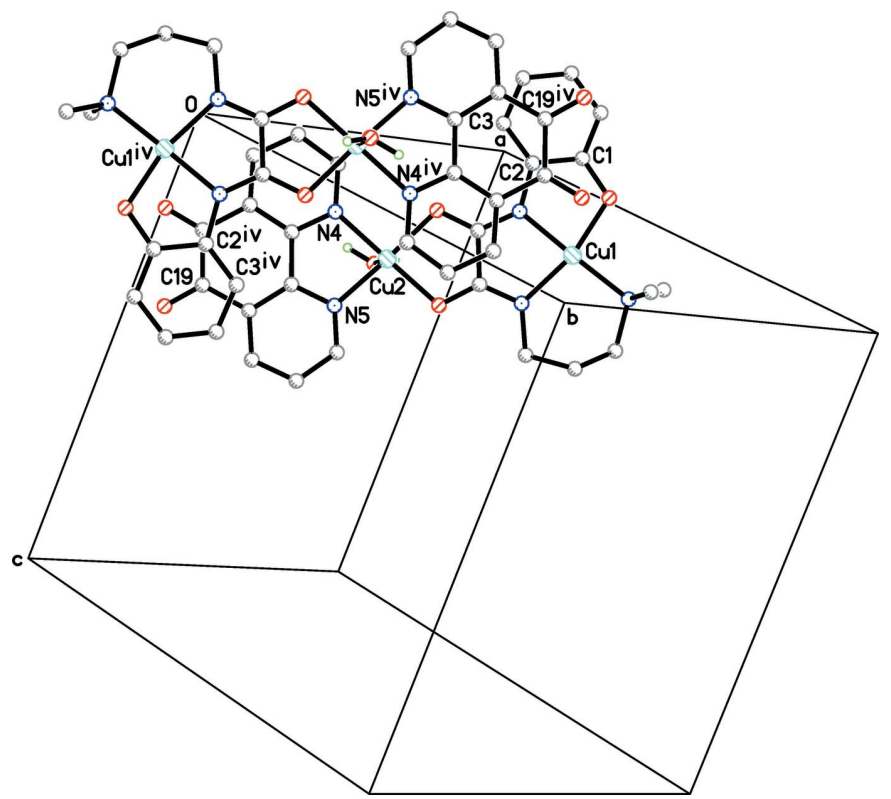

Figure 3

A perspective view of the $\pi-\pi$ stacking interactions viewed perpendicular to the middle ring of the Phdo ligand. $\mathrm{H}$ atoms have been omitted for clarity [symmetry code: (iv) $-x, 1-y,-z]$.

the dimers are assembled by perchlorate anions, generating a wave-like layer parallel to (100). Subsequently, an offset $\pi-\pi$ stacking interaction occurs between the middle aromatic ring of the Phdo ligand of a binuclear unit and the benzene ring of the other unit at $-x, 1-y,-z$ [symmetry code (iv)], and vice versa (Fig. 3). The separations of the overlapped atoms from their opposite rings are $3.191(4)\left(\mathrm{C}^{\text {iv }}\right), 3.211(4)\left(\mathrm{C}^{\text {iv }}\right)$ and 3.252 (4) $\AA$ (C19 $\left.{ }^{\text {iv }}\right)$.

\section{Database survey}

Several $\mathrm{Cu}^{\mathrm{II}}$ complexes of 1,10-phenanthroline-5,6-dione have been reported previously, for example, Chetana et al. (2009); Galet et al. (2005); Saravani et al. (2007); Wang et al. (2013); Yamada et al. (2002) and $\mathrm{Xu}$ et al. (2006).

\section{Synthesis and crystallization}

$N$-[3-(Dimethylamino)propyl]- $N^{\prime}$-2-(oxidophenyl)oxamide $\left(\mathrm{H}_{3}\right.$ Dmapox; Zhang et al., 2013) and 1,10-phenanthroline-5,6dione (Phdo; Dickeson \& Summers, 1970) were prepared by published procedures. The title compound was obtained as follows: A solution of $\mathrm{Cu}\left(\mathrm{ClO}_{4}\right)_{2} \cdot 6 \mathrm{H}_{2} \mathrm{O}(0.0371 \mathrm{~g}, 0.1 \mathrm{mmol})$ in methanol $(5 \mathrm{ml})$ was added dropwise to a solution of $\mathrm{H}_{3}$ Dmapox $(0.0133 \mathrm{~g}, 0.05 \mathrm{mmol})$ and piperidine $(0.0128 \mathrm{~g}$, $0.15 \mathrm{mmol})$ in methanol $(5 \mathrm{ml})$. The solution was stirred continuously for $0.5 \mathrm{~h}$. Then a solution of Phdo $(0.011 \mathrm{~g}$, $0.05 \mathrm{mmol})$ in methanol $(5 \mathrm{ml})$ was added dropwise, and the mixture was stirred continuously at $313 \mathrm{~K}$ for $6 \mathrm{~h}$ and then filtered. Dark-blue crystals of the title compound suitable for X-ray analysis were obtained from the filtrate by slow evaporation at room temperature for $7 \mathrm{~d}$. Yield: $0.026 \mathrm{~g}$
Table 3

Experimental details.

\begin{tabular}{|c|c|}
\hline \multicolumn{2}{|l|}{ Crystal data } \\
\hline Chemical formula & $\begin{array}{l}{\left[\mathrm{Cu}_{2}\left(\mathrm{C}_{13} \mathrm{H}_{16} \mathrm{~N}_{3} \mathrm{O}_{3}\right)\left(\mathrm{C}_{12} \mathrm{H}_{6} \mathrm{~N}_{2} \mathrm{O}_{2}\right)-\right.} \\
\left.\quad\left(\mathrm{H}_{2} \mathrm{O}\right)\right] \mathrm{ClO}_{4} \cdot 0.5 \mathrm{H}_{2} \mathrm{O}\end{array}$ \\
\hline$M_{\mathrm{r}}$ & 726.03 \\
\hline Crystal system, space group & Monoclinic, $P 2_{1} / c$ \\
\hline Temperature $(\mathrm{K})$ & 296 \\
\hline$a, b, c(\AA)$ & $\begin{array}{l}11.7430(6), 17.3066(9) \\
\quad 14.1086(8)\end{array}$ \\
\hline$\beta\left(^{\circ}\right)$ & $98.154(1)$ \\
\hline$V\left(\mathrm{~A}^{3}\right)$ & $2838.3(3)$ \\
\hline$Z$ & 4 \\
\hline Radiation type & Мо $K \alpha$ \\
\hline$\mu\left(\mathrm{mm}^{-1}\right)$ & 1.66 \\
\hline Crystal size $(\mathrm{mm})$ & $0.30 \times 0.12 \times 0.06$ \\
\hline \multicolumn{2}{|l|}{ Data collection } \\
\hline Diffractometer & Bruker APEX area detector \\
\hline Absorption correction & $\begin{array}{l}\text { Multi-scan ( } S A D A B S \text {; Bruker, } \\
\text { 2002) }\end{array}$ \\
\hline$T_{\min }, T_{\max }$ & $0.636,0.907$ \\
\hline $\begin{array}{l}\text { No. of measured, independent and } \\
\text { observed }[I>2 \sigma(I)] \text { reflections }\end{array}$ & $21066,6432,4831$ \\
\hline$R_{\text {int }}$ & 0.054 \\
\hline$(\sin \theta / \lambda)_{\max }\left(\AA^{-1}\right)$ & 0.649 \\
\hline \multicolumn{2}{|l|}{ Refinement } \\
\hline$R\left[F^{2}>2 \sigma\left(F^{2}\right)\right], w R\left(F^{2}\right), S$ & $0.050,0.114,1.05$ \\
\hline No. of reflections & 6432 \\
\hline No. of parameters & 478 \\
\hline No. of restraints & 31 \\
\hline $\mathrm{H}$-atom treatment & H-atom parameters constrained \\
\hline$\Delta \rho_{\max }, \Delta \rho_{\min }\left(\mathrm{e} \AA^{-3}\right)$ & $0.76,-0.48$ \\
\hline
\end{tabular}

Computer programs: SMART and SAINT (Bruker, 2002), SHELXS97, SHELXL97 and $X P$ in SHELXTL (Sheldrick, 2008) and WinGX (Farrugia, 2012).

(71.62\%). Analysis calculated for $\mathrm{Cu}_{2} \mathrm{C}_{25} \mathrm{H}_{25} \mathrm{ClN}_{5} \mathrm{O}_{10.5}$ : C 41.44, H 3.48, N 9.67\%; found: C 42.57, H 3.15, N 9.19\%.

\section{Refinement}

Crystal data, data collection, and refinement details are summarized in Table 3. Disorder occurs for four carbon atoms of the 3-(dimethylamino)propyl group [C10 $A-\mathrm{C} 13 A$, with occupancies of $0.561(11) ; \mathrm{C} 10 B-\mathrm{C} 13 B, 0.439(11)]$, three oxygen atoms of the perchlorate ion [O9A-O11A, 0.646 (8); $\mathrm{O} 9 B-\mathrm{O} 11 B, 0.354(8)]$ and the solvent water molecule $(\mathrm{O} 7 A$, 0.207 (10); O7B, $0.293(10)]$. The occupancies were refined freely except for the sum of atoms $\mathrm{O} 7 A$ and $\mathrm{O} 7 B$ which was fixed at 0.5 . Some restraints on distances (DFIX) and anisotropic displacement parameters (SIMU) were applied to the disordered atoms to avoid unreasonable geometries. The hydrogen atoms of the water molecules were found in a difference Fourier map and then refined as riding. Other $\mathrm{H}$ atoms were placed in calculated positions, with $\mathrm{C}-\mathrm{H}=0.96$ (methyl), 0.97 (methylene) and $0.93 \AA$ (aromatic), and refined using a riding model, with $U_{\text {iso }}(\mathrm{H})=1.2 U_{\text {eq }}(\mathrm{C})$ or 1.5 for methyl groups.

\section{Acknowledgements}

This project was supported by the Fundamental Research Funds for the Central Universities, China (grant No. 
201213020) and the Program for Science and Technology of Shandong Province, China (grant No. 2012GHY11525).

\section{References}

Addison, A. W., Rao, T. N., Reedijk, J., van Rijn, J. \& Verschoor, G. C. (1984). J. Chem. Soc. Dalton Trans. pp. 1349-1356.

Bruker (2002). SMART, SAINT and SADABS. Bruker AXS Inc., Madison, Wisconsin, USA

Calderazzo, F., Pampaloni, G. \& Passarelli, V. (2002). Inorg. Chim. Acta, 330, 136-142.

Chetana, P. R., Rao, R., Roy, M. \& Patra, A. K. (2009). Inorg. Chim. Acta, 362, 4692-4698.

Dickeson, J. E. \& Summers, L. A. (1970). Aust. J. Chem. 23, $1023-$ 1027.

Farrugia, L. J. (2012). J. Appl. Cryst. 45, 849-854.

Galet, A., Muñoz, M. C., Agustí, G., Martínez, V., Gaspar, A. B. \& Real, J. A. (2005). Z. Anorg. Allg. Chem. 631, 1985-1987.

Gao, Z. \& Wang, Y. (2010). Acta Cryst. E66, m1249-m1250.

Girgis, A. Y., Sohn, Y. S. \& Balch, A. L. (1975). Inorg. Chem. 14, 2327-2331.
Li, X.-W., Li, X., Li, Y.-T., Wu, Z.-Y. \& Yan, C.-W. (2012). J. Organomet. Chem. 700, 48-57.

Li, X.-W., Zheng, Y.-J., Li, Y.-T., Wu, Z.-Y. \& Yan, C.-W. (2011). Eur. J. Med. Chem. 46, 3851-3857.

Lu, H.-H., Li, Y.-T., Wu, Z.-Y., Zheng, K. \& Yan, C.-W. (2011). J. Coord. Chem. 64, 1360-1374.

Messori, L., Shaw, J., Camalli, M., Mura, P. \& Marcon, G. (2003). Inorg. Chem. 42, 6166-6168.

Ojima, H. \& Nonoyama, K. (1988). Coord. Chem. Rev. 92, 85-111.

Ruiz, R., Faus, J., Lloret, F., Julve, M. \& Journaux, Y. (1999). Coord. Chem. Rev. 193-195, 1069-1117.

Saravani, H., Rezvani, A. R., Mansouri, G., Rad, A. R. S., Khavasi, H. R. \& Hadadzadeh, H. (2007). Inorg. Chim. Acta, 360, 2829-2834.

Sheldrick, G. M. (2008). Acta Cryst. A64, 112-122.

Wang, X.-L., Gao, Q., Tian, A.-X., Zhao, D. \& Liu, X.-J. (2013). J. Coord. Chem. 66, 358-366.

Xu, G.-J., Kou, Y.-Y., Feng, L., Yan, S.-P., Liao, D.-Z., Jiang, Z.-H. \& Cheng, P. (2006). Appl. Organomet. Chem. 20, 351-356.

Yamada, Y., Sakurai, H., Miyashita, Y., Fujisawa, K. \& Okamoto, K. (2002). Polyhedron, 21, 2143-2147.

Zhang, W.-J., Wang, F., Li, Y.-T. \& Wu, Z.-Y. (2013). Transition Met. Chem. 38, 69-78. 


\section{supporting information}

Acta Cryst. (2015). E71, 667-670 [doi:10.1107/S2056989015009391]

\section{Crystal structure of aqua $\left\{\mu-N-\left[3-\left(\right.\right.\right.$ dimethylamino)propyl]- $N^{\prime}-2$-(oxidophenyl)- oxamidato\}(1,10-phenanthroline-5,6-dione)dicopper(II) perchlorate hemihydrate}

\section{Xin Zhang, Yan-Tuan Li and Zhi-Yong Wu}

\section{Computing details}

Data collection: SMART (Bruker, 2002); cell refinement: SAINT (Bruker, 2002); data reduction: SAINT (Bruker, 2002); program(s) used to solve structure: SHELXS97 (Sheldrick, 2008); program(s) used to refine structure: SHELXL97 (Sheldrick, 2008); molecular graphics: XP in SHELXTL (Sheldrick, 2008); software used to prepare material for publication: $\operatorname{Win} G X$ (Farrugia, 2012).

\section{Aqua $\left\{\mu-N\right.$-[3-(dimethylamino)propyl]- $N^{\prime}-2$-(oxidophenyl)oxamidato\}(1,10-phenanthroline-5,6-}

dione)dicopper(II) perchlorate hemihydrate

Crystal data

$\left[\mathrm{Cu}_{2}\left(\mathrm{C}_{13} \mathrm{H}_{16} \mathrm{~N}_{3} \mathrm{O}_{3}\right)\left(\mathrm{C}_{12} \mathrm{H}_{6} \mathrm{~N}_{2} \mathrm{O}_{2}\right)\right.$

$\left.\left(\mathrm{H}_{2} \mathrm{O}\right)\right] \mathrm{ClO}_{4} \cdot 0.5 \mathrm{H}_{2} \mathrm{O}$

$M_{r}=726.03$

Monoclinic, $P 2_{1} / c$

$a=11.7430(6) \AA$

$b=17.3066(9) \AA$

$c=14.1086(8) \AA$

$\beta=98.154(1)^{\circ}$

$V=2838.3(3) \AA^{3}$

$Z=4$

\section{Data collection}

Bruker APEX area-detector diffractometer

Radiation source: fine-focus sealed tube

Graphite monochromator

$\varphi$ and $\omega$ scans

Absorption correction: multi-scan

(SADABS; Bruker, 2002)

$T_{\min }=0.636, T_{\max }=0.907$

Refinement

Refinement on $F^{2}$

Least-squares matrix: full

$R\left[F^{2}>2 \sigma\left(F^{2}\right)\right]=0.050$

$w R\left(F^{2}\right)=0.114$

$S=1.05$
$F(000)=1476$

$D_{\mathrm{x}}=1.699 \mathrm{Mg} \mathrm{m}^{-3}$

Mo $K \alpha$ radiation, $\lambda=0.71073 \AA$

Cell parameters from 9065 reflections

$\theta=3.2-27.4^{\circ}$

$\mu=1.66 \mathrm{~mm}^{-1}$

$T=296 \mathrm{~K}$

Prism, dark blue

$0.30 \times 0.12 \times 0.06 \mathrm{~mm}$

21066 measured reflections

6432 independent reflections

4831 reflections with $I>2 \sigma(I)$

$R_{\text {int }}=0.054$

$\theta_{\max }=27.5^{\circ}, \theta_{\min }=3.2^{\circ}$

$h=-14 \rightarrow 15$

$k=-22 \rightarrow 22$

$l=-15 \rightarrow 18$

6432 reflections

478 parameters

31 restraints

Primary atom site location: structure-invariant direct methods 
Secondary atom site location: difference Fourier map

Hydrogen site location: inferred from neighbouring sites

$\mathrm{H}$-atom parameters constrained

$$
\begin{aligned}
& w=1 /\left[\sigma^{2}\left(F_{\mathrm{o}}^{2}\right)+(0.0369 P)^{2}+7.4021 P\right] \\
& \text { where } P=\left(F_{\mathrm{o}}^{2}+2 F_{\mathrm{c}}^{2}\right) / 3 \\
& (\Delta / \sigma)_{\max }<0.001 \\
& \Delta \rho_{\max }=0.76 \text { e } \AA^{-3} \\
& \Delta \rho_{\min }=-0.47 \mathrm{e} \AA^{-3}
\end{aligned}
$$

Special details

Geometry. All s.u.'s (except the s.u. in the dihedral angle between two 1.s. planes) are estimated using the full covariance matrix. The cell s.u.'s are taken into account individually in the estimation of s.u.'s in distances, angles and torsion angles; correlations between s.u.'s in cell parameters are only used when they are defined by crystal symmetry. An approximate (isotropic) treatment of cell s.u.'s is used for estimating s.u.'s involving l.s. planes.

Refinement. Refinement of $F^{2}$ against ALL reflections. The weighted $R$-factor $w R$ and goodness of fit $S$ are based on $F^{2}$,

\begin{tabular}{|c|c|c|c|c|c|}
\hline & $x$ & $y$ & $z$ & $U_{\text {iso }} * / U_{\text {eq }}$ & Occ. $(<1)$ \\
\hline $\mathrm{Cu} 1$ & $0.48982(4)$ & $0.62884(2)$ & $0.02261(3)$ & $0.01855(12)$ & \\
\hline $\mathrm{Cu} 2$ & $0.15568(4)$ & $0.46352(3)$ & $0.12248(3)$ & $0.01949(12)$ & \\
\hline $\mathrm{O} 1$ & $0.5443(2)$ & $0.62076(14)$ & $-0.10126(18)$ & $0.0201(5)$ & \\
\hline $\mathrm{O} 2$ & $0.2204(2)$ & $0.48428(14)$ & 0.00502 (19) & $0.0202(5)$ & \\
\hline $\mathrm{O} 3$ & $0.2675(2)$ & $0.53779(14)$ & 0.18328 (19) & $0.0216(6)$ & \\
\hline $\mathrm{O} 4$ & $0.2568(2)$ & $0.35877(15)$ & $0.1685(2)$ & $0.0260(6)$ & \\
\hline $\mathrm{H} 4 \mathrm{~A}$ & 0.3245 & 0.3523 & 0.1580 & $0.07(2)^{*}$ & \\
\hline H4B & 0.2148 & 0.3146 & 0.1550 & $0.052(16)^{*}$ & \\
\hline $\mathrm{O} 5$ & $-0.3086(2)$ & $0.27160(16)$ & $0.1260(2)$ & $0.0326(7)$ & \\
\hline O6 & -0.2449 & $0.30625(18)$ & $0.3165(2)$ & $0.0376(8)$ & \\
\hline N1 & $0.3763(2)$ & $0.55502(16)$ & $-0.0332(2)$ & $0.0174(6)$ & \\
\hline N2 & $0.4133(3)$ & $0.61345(17)$ & $0.1376(2)$ & $0.0191(6)$ & \\
\hline N3 & $0.5927(3)$ & $0.71698(18)$ & $0.0732(2)$ & $0.0255(7)$ & \\
\hline N4 & $0.0213(2)$ & $0.40632(17)$ & $0.0548(2)$ & $0.0176(6)$ & \\
\hline N5 & $0.0705(3)$ & 0.45017 (19) & $0.2338(2)$ & $0.0254(7)$ & \\
\hline $\mathrm{C} 1$ & $0.4728(3)$ & $0.5781(2)$ & $-0.1637(3)$ & $0.0193(8)$ & \\
\hline $\mathrm{C} 2$ & $0.3778(3)$ & 0.54062 (19) & -0.1310 & $0.0176(7)$ & \\
\hline $\mathrm{C} 3$ & $0.2998(3)$ & $0.4979(2)$ & -0.1929 & $0.0214(8)$ & \\
\hline $\mathrm{H} 3$ & 0.2375 & 0.4744 & -0.1706 & $0.026^{*}$ & \\
\hline $\mathrm{C} 4$ & $0.3151(4)$ & $0.4902(2)$ & -0.2881 & $0.0270(9)$ & \\
\hline $\mathrm{H} 4$ & 0.2625 & 0.4623 & -0.3303 & $0.032 *$ & \\
\hline C5 & $0.4095(4)$ & $0.5246(2)$ & -0.3199 & $0.0286(9)$ & \\
\hline H5 & 0.4205 & 0.5186 & -0.3835 & $0.034^{*}$ & \\
\hline C6 & $0.4876(3)$ & $0.5676(2)$ & $-0.2587(3)$ & $0.0249(9)$ & \\
\hline H6 & 0.5506 & 0.5897 & -0.2815 & $0.030 *$ & \\
\hline $\mathrm{C} 7$ & 0.3069 (3) & $0.52992(19)$ & 0.0233 & $0.0170(7)$ & \\
\hline $\mathrm{C} 8$ & $0.3321(3)$ & $0.56215(19)$ & 0.1238 & $0.0178(7)$ & \\
\hline C9 & $0.4368(3)$ & $0.6507(2)$ & $0.2314(3)$ & $0.0253(9)$ & \\
\hline H9A & 0.4864 & 0.6178 & 0.2751 & $0.030 *$ & $0.561(11)$ \\
\hline H9B & 0.3653 & 0.6579 & 0.2573 & $0.030 *$ & $0.561(11)$ \\
\hline
\end{tabular}
conventional $R$-factors $R$ are based on $F$, with $F$ set to zero for negative $F^{2}$. The threshold expression of $F^{2}>2 \sigma\left(F^{2}\right)$ is used only for calculating $R$-factors $(\mathrm{gt})$ etc. and is not relevant to the choice of reflections for refinement. $R$-factors based on $F^{2}$ are statistically about twice as large as those based on $F$, and $R$-factors based on ALL data will be even larger.

Fractional atomic coordinates and isotropic or equivalent isotropic displacement parameters $\left(\AA^{2}\right)$ 


\begin{tabular}{|c|c|c|c|c|c|}
\hline $\mathrm{H} 9 \mathrm{C}$ & 0.3716 & 0.6824 & 0.2419 & $0.030 *$ & $0.439(11)$ \\
\hline H9D & 0.4476 & 0.6116 & 0.2811 & $0.030 *$ & 0.439 (11) \\
\hline $\mathrm{C} 10 \mathrm{~A}$ & $0.4950(7)$ & $0.7291(5)$ & $0.2231(6)$ & 0.0207 (19) & $0.561(11)$ \\
\hline H10A & 0.4427 & 0.7629 & 0.1829 & $0.025^{*}$ & $0.561(11)$ \\
\hline $\mathrm{H} 10 \mathrm{~B}$ & 0.5105 & 0.7525 & 0.2861 & $0.025 *$ & $0.561(11)$ \\
\hline C11A & $0.6069(6)$ & $0.7230(5)$ & $0.1811(4)$ & $0.0213(19)$ & $0.561(11)$ \\
\hline H11A & 0.6536 & 0.7680 & 0.2007 & $0.026^{*}$ & $0.561(11)$ \\
\hline H11B & 0.6485 & 0.6778 & 0.2082 & $0.026^{*}$ & $0.561(11)$ \\
\hline $\mathrm{C} 12 \mathrm{~A}$ & $0.5468(14)$ & $0.7891(6)$ & $0.0286(10)$ & $0.046(4)$ & $0.561(11)$ \\
\hline $\mathrm{H} 12 \mathrm{~A}$ & 0.5380 & 0.7839 & -0.0398 & $0.069^{*}$ & $0.561(11)$ \\
\hline H12B & 0.5990 & 0.8306 & 0.0483 & $0.069 *$ & $0.561(11)$ \\
\hline $\mathrm{H} 12 \mathrm{C}$ & 0.4734 & 0.7999 & 0.0482 & $0.069 *$ & $0.561(11)$ \\
\hline $\mathrm{C} 13 \mathrm{~A}$ & $0.7114(8)$ & $0.7022(15)$ & $0.0540(12)$ & $0.043(5)$ & $0.561(11)$ \\
\hline H13A & 0.7615 & 0.7421 & 0.0830 & $0.065^{*}$ & $0.561(11)$ \\
\hline H13B & 0.7128 & 0.7020 & -0.0139 & $0.065^{*}$ & $0.561(11)$ \\
\hline $\mathrm{H} 13 \mathrm{C}$ & 0.7370 & 0.6530 & 0.0803 & $0.065^{*}$ & $0.561(11)$ \\
\hline $\mathrm{C} 10 \mathrm{~B}$ & $0.5434(12)$ & $0.6994(6)$ & $0.2379(7)$ & $0.032(3)$ & $0.439(11)$ \\
\hline $\mathrm{H} 10 \mathrm{C}$ & 0.6093 & 0.6651 & 0.2436 & $0.038^{*}$ & $0.439(11)$ \\
\hline H10D & 0.5493 & 0.7294 & 0.2965 & $0.038^{*}$ & $0.439(11)$ \\
\hline C11B & $0.5519(11)$ & $0.7548(5)$ & $0.1554(8)$ & $0.036(3)$ & $0.439(11)$ \\
\hline $\mathrm{H} 11 \mathrm{C}$ & 0.4768 & 0.7773 & 0.1348 & $0.043^{*}$ & $0.439(11)$ \\
\hline H11D & 0.6041 & 0.7965 & 0.1778 & $0.043 *$ & 0.439 (11) \\
\hline C12B & $0.5760(13)$ & $0.7781(7)$ & $-0.0015(9)$ & $0.038(4)$ & 0.439 (11) \\
\hline H12D & 0.6208 & 0.8228 & 0.0204 & $0.057^{*}$ & $0.439(11)$ \\
\hline $\mathrm{H} 12 \mathrm{E}$ & 0.4962 & 0.7920 & -0.0139 & $0.057^{*}$ & $0.439(11)$ \\
\hline $\mathrm{H} 12 \mathrm{~F}$ & 0.6005 & 0.7591 & -0.0593 & $0.057^{*}$ & $0.439(11)$ \\
\hline C13B & $0.7162(10)$ & $0.6988(17)$ & $0.0870(15)$ & $0.035(5)$ & $0.439(11)$ \\
\hline H13D & 0.7595 & 0.7447 & 0.1058 & $0.053 *$ & $0.439(11)$ \\
\hline H13E & 0.7375 & 0.6796 & 0.0281 & $0.053 *$ & $0.439(11)$ \\
\hline $\mathrm{H} 13 \mathrm{~F}$ & 0.7323 & 0.6603 & 0.1360 & $0.053 *$ & $0.439(11)$ \\
\hline $\mathrm{C} 14$ & $0.0027(3)$ & $0.3843(2)$ & -0.0364 & $0.0206(8)$ & \\
\hline H14 & 0.0530 & 0.4014 & -0.0773 & $0.025^{*}$ & \\
\hline $\mathrm{C} 15$ & -0.0881 & $0.3370(2)$ & $-0.0734(3)$ & $0.0231(8)$ & \\
\hline H15 & -0.0984 & 0.3228 & -0.1376 & $0.028^{*}$ & \\
\hline $\mathrm{C} 16$ & -0.1632 & $0.3114(2)$ & -0.0128 & $0.0227(8)$ & \\
\hline H16 & -0.2245 & 0.2792 & -0.0355 & $0.027 *$ & \\
\hline $\mathrm{C} 17$ & $-0.1456(3)$ & $0.3344(2)$ & $0.0822(3)$ & 0.0183 & \\
\hline $\mathrm{C} 18$ & -0.2225 & $0.3083(2)$ & $0.1514(3)$ & $0.0234(8)$ & \\
\hline C19 & -0.1890 & $0.3309(2)$ & $0.2573(3)$ & $0.0287(9)$ & \\
\hline $\mathrm{C} 20$ & -0.0860 & $0.3801(2)$ & $0.2846(3)$ & $0.0251(8)$ & \\
\hline $\mathrm{C} 21$ & $-0.0471(4)$ & $0.4002(3)$ & $0.3791(3)$ & $0.0375(11)$ & \\
\hline $\mathrm{H} 21$ & -0.0859 & 0.3833 & 0.4283 & $0.045^{*}$ & \\
\hline $\mathrm{C} 22$ & $0.0503(4)$ & 0.4458 & $0.3987(3)$ & $0.0455(13)$ & \\
\hline $\mathrm{H} 22$ & 0.0783 & 0.4595 & 0.4614 & $0.055^{*}$ & \\
\hline $\mathrm{C} 23$ & $0.1057(4)$ & $0.4706(3)$ & $0.3236(3)$ & $0.0387(11)$ & \\
\hline $\mathrm{H} 23$ & 0.1695 & 0.5027 & 0.3368 & $0.046^{*}$ & \\
\hline $\mathrm{C} 24$ & -0.0232 & $0.4052(2)$ & $0.2139(3)$ & $0.0210(8)$ & \\
\hline $\mathrm{C} 25$ & -0.0521 & $0.3817(2)$ & 0.1138 (3) & $0.0170(7)$ & \\
\hline
\end{tabular}




\begin{tabular}{llllll} 
C11 & $0.04798(9)$ & $0.17375(5)$ & $0.17883(7)$ & $0.0275(2)$ & \\
O8 & $0.0854(3)$ & $0.1132(2)$ & $0.2436(3)$ & $0.0567(10)$ & $0.646(8)$ \\
O9A & $0.0082(6)$ & $0.2341(3)$ & $0.2331(4)$ & $0.054(2)$ & $0.646(8)$ \\
O10A & $-0.0380(7)$ & $0.1519(4)$ & $0.1034(4)$ & $0.060(2)$ & $0.646(8)$ \\
O11A & $0.1412(6)$ & $0.2040(4)$ & $0.1353(5)$ & $0.073(2)$ & $0.354(8)$ \\
O9B & $0.0505(16)$ & $0.1419(9)$ & $0.0878(5)$ & $0.112(6)$ & $0.354(8)$ \\
O10B & $0.1088(10)$ & $0.2417(4)$ & $0.1984(12)$ & $0.094(6)$ & $0.354(8)$ \\
O11B & $-0.0679(5)$ & $0.1910(5)$ & $0.1922(7)$ & $0.037(3)$ & $0.207(10)$ \\
O7A & $0.3514(15)$ & $0.5296(11)$ & $0.4141(15)$ & $0.056(7)$ & $0.207(10)$ \\
H7A & 0.3589 & 0.5251 & 0.3546 & $0.084^{*}$ & $0.207(10)$ \\
H7B & 0.4122 & 0.5110 & 0.4473 & $0.084^{*}$ & $0.293(10)$ \\
O7B & $0.282(2)$ & $0.5932(11)$ & $0.4515(15)$ & $0.146(13)$ & $0.293(10)$ \\
H7C & 0.2845 & 0.5848 & 0.5119 & $0.219^{*}$ & $0.293(10)$ \\
H7D & 0.2685 & 0.6416 & 0.4416 & $0.219^{*}$ & \\
\hline
\end{tabular}

Atomic displacement parameters $\left(\AA^{2}\right)$

\begin{tabular}{|c|c|c|c|c|c|c|}
\hline & $U^{11}$ & $U^{22}$ & $U^{\beta 3}$ & $U^{12}$ & $U^{13}$ & $U^{23}$ \\
\hline $\mathrm{Cu} 1$ & $0.0132(2)$ & $0.0162(2)$ & $0.0274(3)$ & $-0.00272(17)$ & $0.00678(18)$ & $-0.00401(18)$ \\
\hline $\mathrm{Cu} 2$ & $0.0134(2)$ & $0.0205(2)$ & $0.0258(3)$ & $-0.00197(18)$ & $0.00698(18)$ & $-0.00077(19)$ \\
\hline $\mathrm{O} 1$ & $0.0139(12)$ & $0.0220(13)$ & $0.0252(14)$ & $-0.0045(10)$ & $0.0057(11)$ & $-0.0014(11)$ \\
\hline $\mathrm{O} 2$ & $0.0151(12)$ & $0.0197(12)$ & $0.0263(14)$ & $-0.0049(10)$ & $0.0053(11)$ & $-0.0003(11)$ \\
\hline $\mathrm{O} 3$ & $0.0186(13)$ & $0.0209(12)$ & $0.0270(15)$ & $-0.0024(11)$ & $0.0091(11)$ & $-0.0006(11)$ \\
\hline $\mathrm{O} 4$ & $0.0175(14)$ & $0.0236(14)$ & $0.0387(17)$ & $0.0015(11)$ & $0.0103(12)$ & $0.0083(12)$ \\
\hline $\mathrm{O} 5$ & $0.0260(16)$ & $0.0291(15)$ & $0.0463(19)$ & $-0.0087(13)$ & $0.0173(14)$ & $0.0000(14)$ \\
\hline O6 & $0.0327(17)$ & $0.0443(18)$ & $0.0399(18)$ & $-0.0003(14)$ & $0.0185(15)$ & $0.0153(15)$ \\
\hline N1 & $0.0135(14)$ & $0.0147(14)$ & $0.0245(17)$ & $0.0001(12)$ & $0.0041(13)$ & $-0.0010(12)$ \\
\hline N2 & $0.0159(15)$ & $0.0180(15)$ & $0.0235(17)$ & $0.0007(12)$ & $0.0028(13)$ & $-0.0013(13)$ \\
\hline N3 & $0.0198(17)$ & $0.0250(16)$ & $0.0334(19)$ & $-0.0058(14)$ & $0.0091(15)$ & $-0.0092(15)$ \\
\hline N4 & $0.0117(14)$ & $0.0198(15)$ & $0.0220(17)$ & $0.0017(12)$ & $0.0046(12)$ & $0.0032(13)$ \\
\hline N5 & $0.0196(16)$ & $0.0308(17)$ & $0.0268(19)$ & $0.0016(14)$ & $0.0061(14)$ & $-0.0042(15)$ \\
\hline $\mathrm{C} 1$ & $0.0145(17)$ & $0.0147(16)$ & $0.029(2)$ & $0.0046(14)$ & $0.0047(15)$ & $0.0007(15)$ \\
\hline $\mathrm{C} 2$ & $0.0123(16)$ & $0.0141(16)$ & $0.026(2)$ & $0.0015(14)$ & $0.0026(15)$ & $-0.0002(15)$ \\
\hline $\mathrm{C} 3$ & $0.0178(18)$ & $0.0181(17)$ & $0.028(2)$ & $-0.0005(15)$ & $0.0038(16)$ & $-0.0008(15)$ \\
\hline $\mathrm{C} 4$ & $0.024(2)$ & $0.0250(19)$ & $0.031(2)$ & $-0.0017(17)$ & $0.0011(17)$ & $-0.0070(17)$ \\
\hline $\mathrm{C} 5$ & $0.030(2)$ & $0.030(2)$ & $0.028(2)$ & $-0.0003(18)$ & $0.0109(18)$ & $-0.0026(18)$ \\
\hline C6 & $0.0203(19)$ & $0.0229(18)$ & $0.033(2)$ & $0.0003(16)$ & $0.0096(17)$ & $0.0025(17)$ \\
\hline $\mathrm{C} 7$ & $0.0112(16)$ & $0.0127(15)$ & $0.027(2)$ & $0.0016(13)$ & $0.0026(14)$ & $0.0017(14)$ \\
\hline $\mathrm{C} 8$ & $0.0140(17)$ & $0.0151(16)$ & $0.025(2)$ & $0.0023(14)$ & $0.0053(15)$ & $0.0000(14)$ \\
\hline C9 & $0.024(2)$ & $0.0260(19)$ & $0.027(2)$ & $-0.0045(16)$ & $0.0062(17)$ & $-0.0065(16)$ \\
\hline $\mathrm{C} 10 \mathrm{~A}$ & $0.020(4)$ & $0.020(4)$ & $0.023(4)$ & $-0.002(3)$ & $0.005(3)$ & $-0.010(3)$ \\
\hline C11A & $0.018(4)$ & 0.018 (4) & $0.030(4)$ & $0.000(3)$ & $0.007(3)$ & -0.009 \\
\hline C12A & $0.067(10)$ & $0.018(5)$ & $0.050(7)$ & $-0.001(5)$ & $0.001(6)$ & $0.000(5)$ \\
\hline C13A & $0.021(5)$ & $0.060(8)$ & $0.052(11)$ & $-0.024(5)$ & $0.013(5)$ & $-0.036(9)$ \\
\hline $\mathrm{C} 10 \mathrm{~B}$ & $0.033(7)$ & $0.031(6)$ & $0.031(6)$ & $-0.002(5)$ & $0.004(5)$ & $-0.006(5)$ \\
\hline C11B & $0.035(7)$ & $0.014(5)$ & $0.063(8)$ & $-0.002(5)$ & $0.019(6)$ & $-0.016(5)$ \\
\hline C12B & $0.019(7)$ & $0.012(5)$ & $0.075(12)$ & 0.002 (4) & $-0.022(7)$ & $-0.015(6)$ \\
\hline C13B & $0.028(6)$ & $0.036(6)$ & 0.039 (12) & $0.001(5)$ & $-0.006(5)$ & -0.017 (9) \\
\hline
\end{tabular}


supporting information

$\begin{array}{lllllll}\text { C14 } & 0.0168(18) & 0.0226(18) & 0.024(2) & 0.0008(15) & 0.0068(15) & 0.0026(15) \\ \text { C15 } & 0.023(2) & 0.0248(19) & 0.022(2) & 0.0029(16) & 0.0036(16) & 0.0012(16) \\ \text { C16 } & 0.0124(17) & 0.0209(18) & 0.034(2) & 0.0026(15) & 0.0019(16) & 0.0039(16) \\ \text { C17 } & 0.0129(17) & 0.0174(16) & 0.025(2) & 0.0002(14) & 0.0043(15) & 0.0030(15) \\ \text { C18 } & 0.0176(19) & 0.0184(17) & 0.037(2) & 0.0048(15) & 0.0111(17) & 0.0071(16) \\ \text { C19 } & 0.025(2) & 0.030(2) & 0.034(2) & 0.0055(17) & 0.0153(18) & 0.0113(18) \\ \text { C20 } & 0.0212(19) & 0.029(2) & 0.026(2) & 0.0060(17) & 0.0095(16) & 0.0049(17) \\ \text { C21 } & 0.031(2) & 0.059(3) & 0.025(2) & 0.008(2) & 0.0125(19) & 0.002(2) \\ \text { C22 } & 0.032(3) & 0.079(4) & 0.026(2) & -0.001(3) & 0.008(2) & -0.013(2) \\ \text { C23 } & 0.027(2) & 0.058(3) & 0.032(3) & -0.004(2) & 0.0057(19) & -0.013(2) \\ \text { C24 } & 0.0140(17) & 0.0241(18) & 0.026(2) & 0.0037(15) & 0.0046(15) & 0.0021(16) \\ \text { C25 } & 0.0116(16) & 0.0181(17) & 0.0220(19) & 0.0047(14) & 0.0049(14) & 0.0021(14) \\ \text { C11 } & 0.0283(5) & 0.0253(5) & 0.0290(5) & 0.0042(4) & 0.0048(4) & 0.0013(4) \\ \text { O8 } & 0.050(2) & 0.053(2) & 0.067(3) & 0.0233(19) & 0.0091(19) & 0.026(2) \\ \text { O9A } & 0.087(6) & 0.042(3) & 0.035(3) & 0.027(4) & 0.018(3) & -0.001(3) \\ \text { O10A } & 0.075(5) & 0.063(4) & 0.037(4) & -0.009(4) & -0.015(3) & 0.002(3) \\ \text { O11A } & 0.089(5) & 0.066(4) & 0.076(5) & -0.040(4) & 0.056(4) & -0.018(4) \\ \text { O9B } & 0.111(13) & 0.175(15) & 0.048(8) & 0.063(11) & 0.006(8) & -0.056(9) \\ \text { O10B } & 0.049(7) & 0.038(6) & 0.196(16) & -0.019(6) & 0.024(9) & 0.008(9) \\ \text { O11B } & 0.016(4) & 0.043(6) & 0.051(6) & 0.009(4) & 0.001(4) & 0.016(5) \\ \text { O7A } & 0.043(11) & 0.056(13) & 0.073(14) & 0.001(9) & 0.022(9) & 0.000(10) \\ \text { O7B } & 0.27(3) & 0.065(13) & 0.096(16) & -0.036(17) & -0.008(18) & 0.009(11)\end{array}$

Geometric parameters ( $\left.\AA,{ }^{\circ}\right)$

\begin{tabular}{llll}
\hline $\mathrm{Cu} 1-\mathrm{O} 1$ & $1.950(3)$ & $\mathrm{C} 10 \mathrm{~A}-\mathrm{H} 10 \mathrm{~B}$ & 0.9700 \\
$\mathrm{Cu} 1-\mathrm{N} 1$ & $1.932(3)$ & $\mathrm{C} 11 \mathrm{~A}-\mathrm{H} 11 \mathrm{~A}$ & 0.9700 \\
$\mathrm{Cu} 1-\mathrm{N} 2$ & $1.982(3)$ & $\mathrm{C} 11 \mathrm{~A}-\mathrm{H} 11 \mathrm{~B}$ & 0.9700 \\
$\mathrm{Cu} 1-\mathrm{N} 3$ & $2.013(3)$ & $\mathrm{C} 12 \mathrm{~A}-\mathrm{H} 12 \mathrm{~A}$ & 0.9600 \\
$\mathrm{Cu} 2-\mathrm{O} 2$ & $1.952(3)$ & $\mathrm{C} 12 \mathrm{~A}-\mathrm{H} 12 \mathrm{~B}$ & 0.9600 \\
$\mathrm{Cu} 2-\mathrm{O} 3$ & $1.947(3)$ & $\mathrm{C} 12 \mathrm{~A}-\mathrm{H} 12 \mathrm{C}$ & 0.9600 \\
$\mathrm{Cu} 2-\mathrm{O} 4$ & $2.213(3)$ & $\mathrm{C} 13 \mathrm{~A}-\mathrm{H} 13 \mathrm{~A}$ & 0.9600 \\
$\mathrm{Cu} 2-\mathrm{N} 4$ & $1.989(3)$ & $\mathrm{C} 13 \mathrm{~A}-\mathrm{H} 13 \mathrm{~B}$ & 0.9600 \\
$\mathrm{Cu} 2-\mathrm{N} 5$ & $1.991(3)$ & $\mathrm{C} 13 \mathrm{~A}-\mathrm{H} 13 \mathrm{C}$ & 0.9600 \\
$\mathrm{O} 1-\mathrm{C} 1$ & $1.347(4)$ & $\mathrm{C} 10 \mathrm{~B}-\mathrm{C} 11 \mathrm{~B}$ & $1.522(9)$ \\
$\mathrm{O} 2-\mathrm{C} 7$ & $1.284(4)$ & $\mathrm{C} 10 \mathrm{~B}-\mathrm{H} 10 \mathrm{C}$ & 0.9700 \\
$\mathrm{O} 3-\mathrm{C} 8$ & $1.280(4)$ & $\mathrm{C} 10 \mathrm{~B}-\mathrm{H} 10 \mathrm{D}$ & 0.9700 \\
$\mathrm{O} 4-\mathrm{H} 4 \mathrm{~A}$ & 0.8374 & $\mathrm{C} 11 \mathrm{~B}-\mathrm{H} 11 \mathrm{C}$ & 0.9700 \\
$\mathrm{O} 4-\mathrm{H} 4 \mathrm{~B}$ & 0.9146 & $\mathrm{C} 11 \mathrm{~B}-\mathrm{H} 11 \mathrm{D}$ & 0.9700 \\
$\mathrm{O} 5-\mathrm{C} 18$ & $1.205(5)$ & $\mathrm{C} 12 \mathrm{~B}-\mathrm{H} 12 \mathrm{D}$ & 0.9600 \\
$\mathrm{O} 6-\mathrm{C} 19$ & $1.211(5)$ & $\mathrm{C} 12 \mathrm{~B}-\mathrm{H} 12 \mathrm{E}$ & 0.9600 \\
$\mathrm{~N} 1-\mathrm{C} 7$ & $1.293(4)$ & $\mathrm{C} 12 \mathrm{~B}-\mathrm{H} 12 \mathrm{~F}$ & 0.9600 \\
$\mathrm{~N} 1-\mathrm{C} 2$ & $1.405(5)$ & $\mathrm{C} 13 \mathrm{~B}-\mathrm{H} 13 \mathrm{D}$ & 0.9600 \\
$\mathrm{~N} 2-\mathrm{C} 8$ & $1.297(4)$ & $\mathrm{C} 13 \mathrm{~B}-\mathrm{H} 13 \mathrm{E}$ & 0.9600 \\
$\mathrm{~N} 2-\mathrm{C} 9$ & $1.463(5)$ & $\mathrm{C} 13 \mathrm{~B}-\mathrm{H} 13 \mathrm{~F}$ & 0.9600 \\
$\mathrm{~N} 3-\mathrm{C} 12 \mathrm{~A}$ & $1.465(8)$ & $\mathrm{C} 14-\mathrm{C} 15$ & $1.385(5)$ \\
$\mathrm{N} 3-\mathrm{C} 13 \mathrm{~B}$ & $1.470(9)$ & $\mathrm{C} 14-\mathrm{H} 14$ & 0.9300 \\
$\mathrm{~N} 3-\mathrm{C} 11 \mathrm{~B}$ & $1.470(10)$ & $\mathrm{C} 15-\mathrm{C} 16$ & $1.386(5)$ \\
& & &
\end{tabular}




\begin{tabular}{|c|c|c|c|}
\hline $\mathrm{N} 3-\mathrm{C} 13 \mathrm{~A}$ & $1.480(8)$ & $\mathrm{C} 15-\mathrm{H} 15$ & 0.9300 \\
\hline $\mathrm{N} 3-\mathrm{C} 12 \mathrm{~B}$ & $1.487(9)$ & $\mathrm{C} 16-\mathrm{C} 17$ & $1.386(5)$ \\
\hline $\mathrm{N} 3-\mathrm{C} 11 \mathrm{~A}$ & $1.511(6)$ & $\mathrm{C} 16-\mathrm{H} 16$ & 0.9300 \\
\hline $\mathrm{N} 4-\mathrm{C} 14$ & $1.331(5)$ & $\mathrm{C} 17-\mathrm{C} 25$ & $1.390(5)$ \\
\hline $\mathrm{N} 4-\mathrm{C} 25$ & $1.349(4)$ & $\mathrm{C} 17-\mathrm{C} 18$ & $1.491(5)$ \\
\hline $\mathrm{N} 5-\mathrm{C} 23$ & $1.324(5)$ & $\mathrm{C} 18-\mathrm{C} 19$ & $1.541(6)$ \\
\hline $\mathrm{N} 5-\mathrm{C} 24$ & $1.345(5)$ & $\mathrm{C} 19-\mathrm{C} 20$ & $1.484(6)$ \\
\hline $\mathrm{C} 1-\mathrm{C} 6$ & $1.388(5)$ & $\mathrm{C} 20-\mathrm{C} 21$ & $1.391(6)$ \\
\hline $\mathrm{C} 1-\mathrm{C} 2$ & $1.423(5)$ & $\mathrm{C} 20-\mathrm{C} 24$ & $1.391(5)$ \\
\hline $\mathrm{C} 2-\mathrm{C} 3$ & $1.386(5)$ & $\mathrm{C} 21-\mathrm{C} 22$ & $1.384(7)$ \\
\hline $\mathrm{C} 3-\mathrm{C} 4$ & $1.387(6)$ & $\mathrm{C} 21-\mathrm{H} 21$ & 0.9300 \\
\hline $\mathrm{C} 3-\mathrm{H} 3$ & 0.9300 & $\mathrm{C} 22-\mathrm{C} 23$ & $1.388(6)$ \\
\hline $\mathrm{C} 4-\mathrm{C} 5$ & $1.388(5)$ & $\mathrm{C} 22-\mathrm{H} 22$ & 0.9300 \\
\hline $\mathrm{C} 4-\mathrm{H} 4$ & 0.9300 & $\mathrm{C} 23-\mathrm{H} 23$ & 0.9300 \\
\hline $\mathrm{C} 5-\mathrm{C} 6$ & $1.385(6)$ & $\mathrm{C} 24-\mathrm{C} 25$ & $1.463(5)$ \\
\hline $\mathrm{C} 5-\mathrm{H} 5$ & 0.9300 & $\mathrm{Cl1}-\mathrm{O} 10 \mathrm{~B}$ & $1.383(5)$ \\
\hline $\mathrm{C} 6-\mathrm{H} 6$ & 0.9300 & $\mathrm{Cl1}-\mathrm{O} 9 \mathrm{~B}$ & $1.401(5)$ \\
\hline $\mathrm{C} 7-\mathrm{C} 8$ & $1.513(5)$ & $\mathrm{Cl1}-\mathrm{O} 10 \mathrm{~A}$ & $1.411(6)$ \\
\hline $\mathrm{C} 9-\mathrm{C} 10 \mathrm{~B}$ & $1.501(11)$ & $\mathrm{Cl1}-\mathrm{O} 9 \mathrm{~A}$ & $1.413(5)$ \\
\hline $\mathrm{C} 9-\mathrm{C} 10 \mathrm{~A}$ & $1.532(8)$ & $\mathrm{Cl} 1-\mathrm{O} 8$ & $1.419(3)$ \\
\hline C9-H9A & 0.9700 & $\mathrm{C} 11-\mathrm{O} 11 \mathrm{~A}$ & $1.429(4)$ \\
\hline C9- $\mathrm{H} 9 \mathrm{~B}$ & 0.9700 & $\mathrm{C} 11-\mathrm{O} 11 \mathrm{~B}$ & $1.432(5)$ \\
\hline $\mathrm{C} 9-\mathrm{H} 9 \mathrm{C}$ & 0.9700 & O7A-H7A & 0.8596 \\
\hline C9-H9D & 0.9700 & O7A-H7B & 0.8602 \\
\hline $\mathrm{C} 10 \mathrm{~A}-\mathrm{C} 11 \mathrm{~A}$ & $1.519(7)$ & $\mathrm{O} 7 \mathrm{~B}-\mathrm{H} 7 \mathrm{C}$ & 0.8602 \\
\hline $\mathrm{C} 10 \mathrm{~A}-\mathrm{H} 10 \mathrm{~A}$ & 0.9700 & $\mathrm{O} 7 \mathrm{~B}-\mathrm{H} 7 \mathrm{D}$ & 0.8603 \\
\hline $\mathrm{O} 1-\mathrm{Cu} 1-\mathrm{N} 1$ & $83.25(11)$ & $\mathrm{H} 10 \mathrm{~A}-\mathrm{C} 10 \mathrm{~A}-\mathrm{H} 10 \mathrm{~B}$ & 107.8 \\
\hline $\mathrm{O} 1-\mathrm{Cu} 1-\mathrm{N} 2$ & $165.77(11)$ & $\mathrm{N} 3-\mathrm{C} 11 \mathrm{~A}-\mathrm{C} 10 \mathrm{~A}$ & $114.8(6)$ \\
\hline $\mathrm{O} 1-\mathrm{Cu} 1-\mathrm{N} 3$ & $96.65(11)$ & $\mathrm{N} 3-\mathrm{C} 11 \mathrm{~A}-\mathrm{H} 11 \mathrm{~A}$ & 108.6 \\
\hline $\mathrm{N} 1-\mathrm{Cu} 1-\mathrm{N} 2$ & $83.00(13)$ & $\mathrm{C} 10 \mathrm{~A}-\mathrm{C} 11 \mathrm{~A}-\mathrm{H} 11 \mathrm{~A}$ & 108.6 \\
\hline $\mathrm{N} 1-\mathrm{Cu} 1-\mathrm{N} 3$ & $172.12(13)$ & $\mathrm{N} 3-\mathrm{C} 11 \mathrm{~A}-\mathrm{H} 11 \mathrm{~B}$ & 108.6 \\
\hline $\mathrm{N} 2-\mathrm{Cu} 1-\mathrm{N} 3$ & $97.48(13)$ & $\mathrm{C} 10 \mathrm{~A}-\mathrm{C} 11 \mathrm{~A}-\mathrm{H} 11 \mathrm{~B}$ & 108.6 \\
\hline $\mathrm{O} 2-\mathrm{Cu} 2-\mathrm{O} 3$ & $86.16(10)$ & $\mathrm{H} 11 \mathrm{~A}-\mathrm{C} 11 \mathrm{~A}-\mathrm{H} 11 \mathrm{~B}$ & 107.6 \\
\hline $\mathrm{O} 2-\mathrm{Cu} 2-\mathrm{N} 4$ & $93.58(11)$ & $\mathrm{N} 3-\mathrm{C} 12 \mathrm{~A}-\mathrm{H} 12 \mathrm{~A}$ & 109.5 \\
\hline $\mathrm{O} 2-\mathrm{Cu} 2-\mathrm{N} 5$ & $172.09(12)$ & $\mathrm{N} 3-\mathrm{C} 12 \mathrm{~A}-\mathrm{H} 12 \mathrm{~B}$ & 109.5 \\
\hline $\mathrm{O} 3-\mathrm{Cu} 2-\mathrm{N} 4$ & $168.40(11)$ & $\mathrm{N} 3-\mathrm{C} 12 \mathrm{~A}-\mathrm{H} 12 \mathrm{C}$ & 109.5 \\
\hline $\mathrm{O} 3-\mathrm{Cu} 2-\mathrm{N} 5$ & $96.42(12)$ & $\mathrm{N} 3-\mathrm{C} 13 \mathrm{~A}-\mathrm{H} 13 \mathrm{~A}$ & 109.5 \\
\hline $\mathrm{N} 4-\mathrm{Cu} 2-\mathrm{N} 5$ & $82.38(13)$ & $\mathrm{N} 3-\mathrm{C} 13 \mathrm{~A}-\mathrm{H} 13 \mathrm{~B}$ & 109.5 \\
\hline $\mathrm{O} 4-\mathrm{Cu} 2-\mathrm{O} 2$ & $98.20(10)$ & $\mathrm{N} 3-\mathrm{C} 13 \mathrm{~A}-\mathrm{H} 13 \mathrm{C}$ & 109.5 \\
\hline $\mathrm{O} 4-\mathrm{Cu} 2-\mathrm{O} 3$ & $96.39(11)$ & $\mathrm{C} 9-\mathrm{C} 10 \mathrm{~B}-\mathrm{C} 11 \mathrm{~B}$ & $116.9(9)$ \\
\hline $\mathrm{O} 4-\mathrm{Cu} 2-\mathrm{N} 4$ & $95.12(11)$ & $\mathrm{C} 9-\mathrm{C} 10 \mathrm{~B}-\mathrm{H} 10 \mathrm{C}$ & 108.1 \\
\hline $\mathrm{O} 4-\mathrm{Cu} 2-\mathrm{N} 5$ & $88.97(12)$ & $\mathrm{C} 11 \mathrm{~B}-\mathrm{C} 10 \mathrm{~B}-\mathrm{H} 10 \mathrm{C}$ & 108.1 \\
\hline $\mathrm{C} 1-\mathrm{O} 1-\mathrm{Cu} 1$ & $111.8(2)$ & C9-C10B-H10D & 108.1 \\
\hline $\mathrm{C} 7-\mathrm{O} 2-\mathrm{Cu} 2$ & $109.6(2)$ & $\mathrm{C} 11 \mathrm{~B}-\mathrm{C} 10 \mathrm{~B}-\mathrm{H} 10 \mathrm{D}$ & 108.1 \\
\hline $\mathrm{C} 8-\mathrm{O} 3-\mathrm{Cu} 2$ & $110.7(2)$ & $\mathrm{H} 10 \mathrm{C}-\mathrm{C} 10 \mathrm{~B}-\mathrm{H} 10 \mathrm{D}$ & 107.3 \\
\hline $\mathrm{Cu} 2-\mathrm{O} 4-\mathrm{H} 4 \mathrm{~A}$ & 122.8 & $\mathrm{~N} 3-\mathrm{C} 11 \mathrm{~B}-\mathrm{C} 10 \mathrm{~B}$ & $112.7(9)$ \\
\hline $\mathrm{Cu} 2-\mathrm{O} 4-\mathrm{H} 4 \mathrm{~B}$ & 111.8 & $\mathrm{~N} 3-\mathrm{C} 11 \mathrm{~B}-\mathrm{H} 11 \mathrm{C}$ & 109.0 \\
\hline
\end{tabular}




\begin{tabular}{|c|c|c|c|}
\hline $\mathrm{H} 4 \mathrm{~A}-\mathrm{O} 4-\mathrm{H} 4 \mathrm{~B}$ & 110.4 & $\mathrm{C} 10 \mathrm{~B}-\mathrm{C} 11 \mathrm{~B}-\mathrm{H} 11 \mathrm{C}$ & 109.0 \\
\hline $\mathrm{C} 7-\mathrm{N} 1-\mathrm{C} 2$ & $130.0(3)$ & N3-C11B-H11D & 109.0 \\
\hline $\mathrm{C} 7-\mathrm{N} 1-\mathrm{Cu} 1$ & $115.4(3)$ & $\mathrm{C} 10 \mathrm{~B}-\mathrm{C} 11 \mathrm{~B}-\mathrm{H} 11 \mathrm{D}$ & 109.0 \\
\hline $\mathrm{C} 2-\mathrm{N} 1-\mathrm{Cu} 1$ & $114.4(2)$ & $\mathrm{H} 11 \mathrm{C}-\mathrm{C} 11 \mathrm{~B}-\mathrm{H} 11 \mathrm{D}$ & 107.8 \\
\hline $\mathrm{C} 8-\mathrm{N} 2-\mathrm{C} 9$ & $118.6(3)$ & $\mathrm{N} 3-\mathrm{C} 12 \mathrm{~B}-\mathrm{H} 12 \mathrm{D}$ & 109.5 \\
\hline $\mathrm{C} 8-\mathrm{N} 2-\mathrm{Cu} 1$ & $112.2(2)$ & $\mathrm{N} 3-\mathrm{C} 12 \mathrm{~B}-\mathrm{H} 12 \mathrm{E}$ & 109.5 \\
\hline $\mathrm{C} 9-\mathrm{N} 2-\mathrm{Cu} 1$ & $129.2(2)$ & $\mathrm{H} 12 \mathrm{D}-\mathrm{C} 12 \mathrm{~B}-\mathrm{H} 12 \mathrm{E}$ & 109.5 \\
\hline $\mathrm{C} 13 \mathrm{~B}-\mathrm{N} 3-\mathrm{C} 11 \mathrm{~B}$ & $114.7(10)$ & $\mathrm{N} 3-\mathrm{C} 12 \mathrm{~B}-\mathrm{H} 12 \mathrm{~F}$ & 109.5 \\
\hline $\mathrm{C} 12 \mathrm{~A}-\mathrm{N} 3-\mathrm{C} 13 \mathrm{~A}$ & $111.5(11)$ & $\mathrm{H} 12 \mathrm{D}-\mathrm{C} 12 \mathrm{~B}-\mathrm{H} 12 \mathrm{~F}$ & 109.5 \\
\hline $\mathrm{C} 13 \mathrm{~B}-\mathrm{N} 3-\mathrm{C} 12 \mathrm{~B}$ & $105.8(12)$ & $\mathrm{H} 12 \mathrm{E}-\mathrm{C} 12 \mathrm{~B}-\mathrm{H} 12 \mathrm{~F}$ & 109.5 \\
\hline $\mathrm{C} 11 \mathrm{~B}-\mathrm{N} 3-\mathrm{C} 12 \mathrm{~B}$ & $102.6(7)$ & N3-C13B-H13D & 109.5 \\
\hline $\mathrm{C} 12 \mathrm{~A}-\mathrm{N} 3-\mathrm{C} 11 \mathrm{~A}$ & $110.8(6)$ & N3 $-\mathrm{C} 13 \mathrm{~B}-\mathrm{H} 13 \mathrm{E}$ & 109.5 \\
\hline $\mathrm{C} 13 \mathrm{~A}-\mathrm{N} 3-\mathrm{C} 11 \mathrm{~A}$ & $103.0(7)$ & $\mathrm{H} 13 \mathrm{D}-\mathrm{C} 13 \mathrm{~B}-\mathrm{H} 13 \mathrm{E}$ & 109.5 \\
\hline $\mathrm{C} 12 \mathrm{~A}-\mathrm{N} 3-\mathrm{Cu} 1$ & $109.1(7)$ & $\mathrm{N} 3-\mathrm{C} 13 \mathrm{~B}-\mathrm{H} 13 \mathrm{~F}$ & 109.5 \\
\hline $\mathrm{C} 13 \mathrm{~B}-\mathrm{N} 3-\mathrm{Cu} 1$ & $114.6(12)$ & $\mathrm{H} 13 \mathrm{D}-\mathrm{C} 13 \mathrm{~B}-\mathrm{H} 13 \mathrm{~F}$ & 109.5 \\
\hline $\mathrm{C} 11 \mathrm{~B}-\mathrm{N} 3-\mathrm{Cu} 1$ & $111.8(4)$ & $\mathrm{H} 13 \mathrm{E}-\mathrm{C} 13 \mathrm{~B}-\mathrm{H} 13 \mathrm{~F}$ & 109.5 \\
\hline $\mathrm{C} 13 \mathrm{~A}-\mathrm{N} 3-\mathrm{Cu} 1$ & $109.7(9)$ & $\mathrm{N} 4-\mathrm{C} 14-\mathrm{C} 15$ & $123.0(3)$ \\
\hline $\mathrm{C} 12 \mathrm{~B}-\mathrm{N} 3-\mathrm{Cu} 1$ & $106.0(6)$ & $\mathrm{N} 4-\mathrm{C} 14-\mathrm{H} 14$ & 118.5 \\
\hline $\mathrm{C} 11 \mathrm{~A}-\mathrm{N} 3-\mathrm{Cu} 1$ & $112.6(3)$ & $\mathrm{C} 15-\mathrm{C} 14-\mathrm{H} 14$ & 118.5 \\
\hline $\mathrm{C} 14-\mathrm{N} 4-\mathrm{C} 25$ & $118.3(3)$ & $\mathrm{C} 14-\mathrm{C} 15-\mathrm{C} 16$ & $118.7(4)$ \\
\hline $\mathrm{C} 14-\mathrm{N} 4-\mathrm{Cu} 2$ & $128.1(2)$ & $\mathrm{C} 14-\mathrm{C} 15-\mathrm{H} 15$ & 120.6 \\
\hline $\mathrm{C} 25-\mathrm{N} 4-\mathrm{Cu} 2$ & $113.2(2)$ & $\mathrm{C} 16-\mathrm{C} 15-\mathrm{H} 15$ & 120.6 \\
\hline $\mathrm{C} 23-\mathrm{N} 5-\mathrm{C} 24$ & $119.3(4)$ & $\mathrm{C} 17-\mathrm{C} 16-\mathrm{C} 15$ & $118.9(4)$ \\
\hline $\mathrm{C} 23-\mathrm{N} 5-\mathrm{Cu} 2$ & $126.9(3)$ & $\mathrm{C} 17-\mathrm{C} 16-\mathrm{H} 16$ & 120.6 \\
\hline $\mathrm{C} 24-\mathrm{N} 5-\mathrm{Cu} 2$ & $113.0(3)$ & $\mathrm{C} 15-\mathrm{C} 16-\mathrm{H} 16$ & 120.6 \\
\hline $\mathrm{O} 1-\mathrm{C} 1-\mathrm{C} 6$ & $123.5(3)$ & $\mathrm{C} 16-\mathrm{C} 17-\mathrm{C} 25$ & $118.8(3)$ \\
\hline $\mathrm{O} 1-\mathrm{C} 1-\mathrm{C} 2$ & $118.8(3)$ & $\mathrm{C} 16-\mathrm{C} 17-\mathrm{C} 18$ & $121.7(3)$ \\
\hline $\mathrm{C} 6-\mathrm{C} 1-\mathrm{C} 2$ & $117.6(3)$ & $\mathrm{C} 25-\mathrm{C} 17-\mathrm{C} 18$ & $119.5(3)$ \\
\hline $\mathrm{C} 3-\mathrm{C} 2-\mathrm{N} 1$ & $127.9(3)$ & $\mathrm{O} 5-\mathrm{C} 18-\mathrm{C} 17$ & $121.8(4)$ \\
\hline $\mathrm{C} 3-\mathrm{C} 2-\mathrm{C} 1$ & $121.2(3)$ & $\mathrm{O} 5-\mathrm{C} 18-\mathrm{C} 19$ & $120.6(3)$ \\
\hline $\mathrm{N} 1-\mathrm{C} 2-\mathrm{C} 1$ & $110.9(3)$ & $\mathrm{C} 17-\mathrm{C} 18-\mathrm{C} 19$ & $117.6(3)$ \\
\hline $\mathrm{C} 2-\mathrm{C} 3-\mathrm{C} 4$ & $119.8(3)$ & $\mathrm{O} 6-\mathrm{C} 19-\mathrm{C} 20$ & $121.7(4)$ \\
\hline $\mathrm{C} 2-\mathrm{C} 3-\mathrm{H} 3$ & 120.1 & $\mathrm{O} 6-\mathrm{C} 19-\mathrm{C} 18$ & $119.4(4)$ \\
\hline $\mathrm{C} 4-\mathrm{C} 3-\mathrm{H} 3$ & 120.1 & $\mathrm{C} 20-\mathrm{C} 19-\mathrm{C} 18$ & $118.9(3)$ \\
\hline $\mathrm{C} 3-\mathrm{C} 4-\mathrm{C} 5$ & $119.4(4)$ & $\mathrm{C} 21-\mathrm{C} 20-\mathrm{C} 24$ & $118.3(4)$ \\
\hline $\mathrm{C} 3-\mathrm{C} 4-\mathrm{H} 4$ & 120.3 & $\mathrm{C} 21-\mathrm{C} 20-\mathrm{C} 19$ & $122.5(4)$ \\
\hline $\mathrm{C} 5-\mathrm{C} 4-\mathrm{H} 4$ & 120.3 & $\mathrm{C} 24-\mathrm{C} 20-\mathrm{C} 19$ & $119.2(4)$ \\
\hline $\mathrm{C} 6-\mathrm{C} 5-\mathrm{C} 4$ & $121.2(4)$ & $\mathrm{C} 22-\mathrm{C} 21-\mathrm{C} 20$ & $118.9(4)$ \\
\hline $\mathrm{C} 6-\mathrm{C} 5-\mathrm{H} 5$ & 119.4 & $\mathrm{C} 22-\mathrm{C} 21-\mathrm{H} 21$ & 120.5 \\
\hline $\mathrm{C} 4-\mathrm{C} 5-\mathrm{H} 5$ & 119.4 & $\mathrm{C} 20-\mathrm{C} 21-\mathrm{H} 21$ & 120.5 \\
\hline $\mathrm{C} 5-\mathrm{C} 6-\mathrm{C} 1$ & $120.7(4)$ & $\mathrm{C} 21-\mathrm{C} 22-\mathrm{C} 23$ & $119.2(4)$ \\
\hline $\mathrm{C} 5-\mathrm{C} 6-\mathrm{H} 6$ & 119.7 & $\mathrm{C} 21-\mathrm{C} 22-\mathrm{H} 22$ & 120.4 \\
\hline $\mathrm{C} 1-\mathrm{C} 6-\mathrm{H} 6$ & 119.7 & $\mathrm{C} 23-\mathrm{C} 22-\mathrm{H} 22$ & 120.4 \\
\hline $\mathrm{O} 2-\mathrm{C} 7-\mathrm{N} 1$ & $129.3(4)$ & $\mathrm{N} 5-\mathrm{C} 23-\mathrm{C} 22$ & $122.1(4)$ \\
\hline $\mathrm{O} 2-\mathrm{C} 7-\mathrm{C} 8$ & $117.3(3)$ & $\mathrm{N} 5-\mathrm{C} 23-\mathrm{H} 23$ & 119.0 \\
\hline $\mathrm{N} 1-\mathrm{C} 7-\mathrm{C} 8$ & $113.4(3)$ & $\mathrm{C} 22-\mathrm{C} 23-\mathrm{H} 23$ & 119.0 \\
\hline $\mathrm{O} 3-\mathrm{C} 8-\mathrm{N} 2$ & $128.1(3)$ & $\mathrm{N} 5-\mathrm{C} 24-\mathrm{C} 20$ & $122.1(4)$ \\
\hline $\mathrm{O} 3-\mathrm{C} 8-\mathrm{C} 7$ & $116.0(3)$ & $\mathrm{N} 5-\mathrm{C} 24-\mathrm{C} 25$ & $115.7(3)$ \\
\hline
\end{tabular}




\begin{tabular}{|c|c|}
\hline $\mathrm{N} 2-\mathrm{C} 8-\mathrm{C} 7$ & $115.8(3)$ \\
\hline $\mathrm{N} 2-\mathrm{C} 9-\mathrm{C} 10 \mathrm{~B}$ & $110.6(5)$ \\
\hline $\mathrm{N} 2-\mathrm{C} 9-\mathrm{C} 10 \mathrm{~A}$ & $110.5(4)$ \\
\hline $\mathrm{N} 2-\mathrm{C} 9-\mathrm{H} 9 \mathrm{~A}$ & 109.6 \\
\hline $\mathrm{C} 10 \mathrm{~A}-\mathrm{C} 9-\mathrm{H} 9 \mathrm{~A}$ & 109.6 \\
\hline $\mathrm{N} 2-\mathrm{C} 9-\mathrm{H} 9 \mathrm{~B}$ & 109.6 \\
\hline $\mathrm{C} 10 \mathrm{~A}-\mathrm{C} 9-\mathrm{H} 9 \mathrm{~B}$ & 109.6 \\
\hline $\mathrm{H} 9 \mathrm{~A}-\mathrm{C} 9-\mathrm{H} 9 \mathrm{~B}$ & 108.1 \\
\hline $\mathrm{N} 2-\mathrm{C} 9-\mathrm{H} 9 \mathrm{C}$ & 109.6 \\
\hline $\mathrm{C} 10 \mathrm{~B}-\mathrm{C} 9-\mathrm{H} 9 \mathrm{C}$ & 109.9 \\
\hline N2-C9-H9D & 109.6 \\
\hline $\mathrm{C} 10 \mathrm{~B}-\mathrm{C} 9-\mathrm{H} 9 \mathrm{D}$ & 109.0 \\
\hline $\mathrm{H} 9 \mathrm{C}-\mathrm{C} 9-\mathrm{H} 9 \mathrm{D}$ & 108.1 \\
\hline $\mathrm{C} 11 \mathrm{~A}-\mathrm{C} 10 \mathrm{~A}-\mathrm{C} 9$ & $112.8(6)$ \\
\hline $\mathrm{C} 11 \mathrm{~A}-\mathrm{C} 10 \mathrm{~A}-\mathrm{H} 10 \mathrm{~A}$ & 109.0 \\
\hline $\mathrm{C} 9-\mathrm{C} 10 \mathrm{~A}-\mathrm{H} 10 \mathrm{~A}$ & 109.0 \\
\hline $\mathrm{C} 11 \mathrm{~A}-\mathrm{C} 10 \mathrm{~A}-\mathrm{H} 10 \mathrm{~B}$ & 109.0 \\
\hline $\mathrm{C} 9-\mathrm{C} 10 \mathrm{~A}-\mathrm{H} 10 \mathrm{~B}$ & 109.0 \\
\hline $\mathrm{N} 1-\mathrm{Cu} 1-\mathrm{O} 1-\mathrm{C} 1$ & $7.0(2)$ \\
\hline $\mathrm{N} 2-\mathrm{Cu} 1-\mathrm{O} 1-\mathrm{C} 1$ & $22.0(6)$ \\
\hline $\mathrm{N} 3-\mathrm{Cu} 1-\mathrm{O} 1-\mathrm{C} 1$ & $-165.1(2)$ \\
\hline $\mathrm{O} 3-\mathrm{Cu} 2-\mathrm{O} 2-\mathrm{C} 7$ & $4.6(2)$ \\
\hline $\mathrm{N} 4-\mathrm{Cu} 2-\mathrm{O} 2-\mathrm{C} 7$ & $173.0(2)$ \\
\hline $\mathrm{O} 4-\mathrm{Cu} 2-\mathrm{O} 2-\mathrm{C} 7$ & $-91.3(2)$ \\
\hline $\mathrm{O} 2-\mathrm{Cu} 2-\mathrm{O} 3-\mathrm{C} 8$ & $-2.9(2)$ \\
\hline $\mathrm{N} 4-\mathrm{Cu} 2-\mathrm{O} 3-\mathrm{C} 8$ & $-92.0(6)$ \\
\hline $\mathrm{N} 5-\mathrm{Cu} 2-\mathrm{O} 3-\mathrm{C} 8$ & $-175.4(2)$ \\
\hline $\mathrm{O} 4-\mathrm{Cu} 2-\mathrm{O} 3-\mathrm{C} 8$ & $94.9(2)$ \\
\hline $\mathrm{O} 1-\mathrm{Cu} 1-\mathrm{N} 1-\mathrm{C} 7$ & $176.1(3)$ \\
\hline $\mathrm{N} 2-\mathrm{Cu} 1-\mathrm{N} 1-\mathrm{C} 7$ & $-0.2(3)$ \\
\hline $\mathrm{O} 1-\mathrm{Cu} 1-\mathrm{N} 1-\mathrm{C} 2$ & $-7.7(2)$ \\
\hline $\mathrm{N} 2-\mathrm{Cu} 1-\mathrm{N} 1-\mathrm{C} 2$ & $176.0(2)$ \\
\hline $\mathrm{N} 1-\mathrm{Cu} 1-\mathrm{N} 2-\mathrm{C} 8$ & $2.7(2)$ \\
\hline $\mathrm{O} 1-\mathrm{Cu} 1-\mathrm{N} 2-\mathrm{C} 8$ & $-12.3(6)$ \\
\hline $\mathrm{N} 3-\mathrm{Cu} 1-\mathrm{N} 2-\mathrm{C} 8$ & $174.8(2)$ \\
\hline $\mathrm{N} 1-\mathrm{Cu} 1-\mathrm{N} 2-\mathrm{C} 9$ & $-177.8(3)$ \\
\hline $\mathrm{O} 1-\mathrm{Cu} 1-\mathrm{N} 2-\mathrm{C} 9$ & $167.2(4)$ \\
\hline $\mathrm{N} 3-\mathrm{Cu} 1-\mathrm{N} 2-\mathrm{C} 9$ & $-5.7(3)$ \\
\hline $\mathrm{O} 1-\mathrm{Cu} 1-\mathrm{N} 3-\mathrm{C} 12 \mathrm{~A}$ & $76.8(6)$ \\
\hline $\mathrm{N} 2-\mathrm{Cu} 1-\mathrm{N} 3-\mathrm{C} 12 \mathrm{~A}$ & $-105.0(6)$ \\
\hline $\mathrm{O} 1-\mathrm{Cu} 1-\mathrm{N} 3-\mathrm{C} 13 \mathrm{~B}$ & $-64.5(10)$ \\
\hline $\mathrm{N} 2-\mathrm{Cu} 1-\mathrm{N} 3-\mathrm{C} 13 \mathrm{~B}$ & $113.8(10)$ \\
\hline $\mathrm{O} 1-\mathrm{Cu} 1-\mathrm{N} 3-\mathrm{C} 11 \mathrm{~B}$ & $162.9(5)$ \\
\hline $\mathrm{N} 2-\mathrm{Cu} 1-\mathrm{N} 3-\mathrm{C} 11 \mathrm{~B}$ & $-18.9(6)$ \\
\hline $\mathrm{O} 1-\mathrm{Cu} 1-\mathrm{N} 3-\mathrm{C} 13 \mathrm{~A}$ & $-45.6(9)$ \\
\hline $\mathrm{N} 2-\mathrm{Cu} 1-\mathrm{N} 3-\mathrm{C} 13 \mathrm{~A}$ & $132.6(9)$ \\
\hline $\mathrm{O} 1-\mathrm{Cu} 1-\mathrm{N} 3-\mathrm{C} 12 \mathrm{~B}$ & $51.8(6)$ \\
\hline
\end{tabular}

\begin{tabular}{|c|c|}
\hline $\mathrm{C} 20-\mathrm{C} 24-\mathrm{C} 25$ & $122.1(3)$ \\
\hline $\mathrm{N} 4-\mathrm{C} 25-\mathrm{C} 17$ & $122.2(3)$ \\
\hline $\mathrm{N} 4-\mathrm{C} 25-\mathrm{C} 24$ & $115.2(3)$ \\
\hline $\mathrm{C} 17-\mathrm{C} 25-\mathrm{C} 24$ & $122.5(3)$ \\
\hline $\mathrm{O} 10 \mathrm{~B}-\mathrm{Cl1}-\mathrm{O} 9 \mathrm{~B}$ & $116.1(10)$ \\
\hline $\mathrm{O} 10 \mathrm{~A}-\mathrm{Cl1}-\mathrm{O} 9 \mathrm{~A}$ & $110.6(4)$ \\
\hline $\mathrm{O} 10 \mathrm{~B}-\mathrm{Cl1}-\mathrm{O} 8$ & $113.3(6)$ \\
\hline $\mathrm{O} 9 \mathrm{~B}-\mathrm{Cl1}-\mathrm{O} 8$ & $104.8(5)$ \\
\hline $\mathrm{O} 10 \mathrm{~A}-\mathrm{Cl1}-\mathrm{O} 8$ & $114.2(3)$ \\
\hline $\mathrm{O} 9 \mathrm{~A}-\mathrm{Cl1}-\mathrm{O} 8$ & $107.0(3)$ \\
\hline $\mathrm{O} 10 \mathrm{~A}-\mathrm{C} 11-\mathrm{O} 11 \mathrm{~A}$ & $106.5(4)$ \\
\hline $\mathrm{O} 9 \mathrm{~A}-\mathrm{Cl1}-\mathrm{O} 11 \mathrm{~A}$ & $107.2(4)$ \\
\hline $\mathrm{O} 8-\mathrm{Cl1}-\mathrm{O} 11 \mathrm{~A}$ & $111.2(4)$ \\
\hline $\mathrm{O} 10 \mathrm{~B}-\mathrm{Cl1}-\mathrm{O} 11 \mathrm{~B}$ & $105.7(7)$ \\
\hline $\mathrm{O} 9 \mathrm{~B}-\mathrm{Cl1}-\mathrm{O} 11 \mathrm{~B}$ & $110.3(8)$ \\
\hline $\mathrm{O} 8-\mathrm{Cl1}-\mathrm{O} 11 \mathrm{~B}$ & $106.4(4)$ \\
\hline $\mathrm{H} 7 \mathrm{~A}-\mathrm{O} 7 \mathrm{~A}-\mathrm{H} 7 \mathrm{~B}$ & 107.8 \\
\hline $\mathrm{H} 7 \mathrm{C}-\mathrm{O} 7 \mathrm{~B}-\mathrm{H} 7 \mathrm{D}$ & 107.8 \\
\hline $\mathrm{Cu} 2-\mathrm{O} 3-\mathrm{C} 8-\mathrm{N} 2$ & $177.6(3)$ \\
\hline $\mathrm{Cu} 2-\mathrm{O} 3-\mathrm{C} 8-\mathrm{C} 7$ & $0.8(4)$ \\
\hline $\mathrm{C} 9-\mathrm{N} 2-\mathrm{C} 8-\mathrm{O} 3$ & $-0.8(6)$ \\
\hline $\mathrm{Cu} 1-\mathrm{N} 2-\mathrm{C} 8-\mathrm{O} 3$ & $178.8(3)$ \\
\hline $\mathrm{C} 9-\mathrm{N} 2-\mathrm{C} 8-\mathrm{C} 7$ & 176.0 \\
\hline $\mathrm{Cu} 1-\mathrm{N} 2-\mathrm{C} 8-\mathrm{C} 7$ & $-4.4(4)$ \\
\hline $\mathrm{O} 2-\mathrm{C} 7-\mathrm{C} 8-\mathrm{O} 3$ & $3.3(5)$ \\
\hline $\mathrm{N} 1-\mathrm{C} 7-\mathrm{C} 8-\mathrm{O} 3$ & $-178.5(3)$ \\
\hline $\mathrm{O} 2-\mathrm{C} 7-\mathrm{C} 8-\mathrm{N} 2$ & $-174.0(3)$ \\
\hline $\mathrm{N} 1-\mathrm{C} 7-\mathrm{C} 8-\mathrm{N} 2$ & $4.3(4)$ \\
\hline $\mathrm{C} 8-\mathrm{N} 2-\mathrm{C} 9-\mathrm{C} 10 \mathrm{~B}$ & $172.0(6)$ \\
\hline $\mathrm{Cu} 1-\mathrm{N} 2-\mathrm{C} 9-\mathrm{C} 10 \mathrm{~B}$ & $-7.5(7)$ \\
\hline $\mathrm{C} 8-\mathrm{N} 2-\mathrm{C} 9-\mathrm{C} 10 \mathrm{~A}$ & $-156.4(5)$ \\
\hline $\mathrm{Cu} 1-\mathrm{N} 2-\mathrm{C} 9-\mathrm{C} 10 \mathrm{~A}$ & $24.1(6)$ \\
\hline $\mathrm{N} 2-\mathrm{C} 9-\mathrm{C} 10 \mathrm{~A}-\mathrm{C} 11 \mathrm{~A}$ & $-58.1(8)$ \\
\hline $\mathrm{C} 12 \mathrm{~A}-\mathrm{N} 3-\mathrm{C} 11 \mathrm{~A}-\mathrm{C} 10 \mathrm{~A}$ & $67.3(10)$ \\
\hline $\mathrm{C} 13 \mathrm{~A}-\mathrm{N} 3-\mathrm{C} 11 \mathrm{~A}-\mathrm{C} 10 \mathrm{~A}$ & $-173.3(12)$ \\
\hline $\mathrm{Cu} 1-\mathrm{N} 3-\mathrm{C} 11 \mathrm{~A}-\mathrm{C} 10 \mathrm{~A}$ & $-55.2(7)$ \\
\hline $\mathrm{C} 9-\mathrm{C} 10 \mathrm{~A}-\mathrm{C} 11 \mathrm{~A}-\mathrm{N} 3$ & $80.9(9)$ \\
\hline $\mathrm{N} 2-\mathrm{C} 9-\mathrm{C} 10 \mathrm{~B}-\mathrm{C} 11 \mathrm{~B}$ & $48.9(12)$ \\
\hline $\mathrm{C} 13 \mathrm{~B}-\mathrm{N} 3-\mathrm{C} 11 \mathrm{~B}-\mathrm{C} 10 \mathrm{~B}$ & $-74.4(16)$ \\
\hline $\mathrm{C} 12 \mathrm{~B}-\mathrm{N} 3-\mathrm{C} 11 \mathrm{~B}-\mathrm{C} 10 \mathrm{~B}$ & $171.4(11)$ \\
\hline $\mathrm{Cu} 1-\mathrm{N} 3-\mathrm{C} 11 \mathrm{~B}-\mathrm{C} 10 \mathrm{~B}$ & $58.2(11)$ \\
\hline $\mathrm{C} 9-\mathrm{C} 10 \mathrm{~B}-\mathrm{C} 11 \mathrm{~B}-\mathrm{N} 3$ & $-82.3(14)$ \\
\hline $\mathrm{C} 25-\mathrm{N} 4-\mathrm{C} 14-\mathrm{C} 15$ & $-0.3(5)$ \\
\hline $\mathrm{Cu} 2-\mathrm{N} 4-\mathrm{C} 14-\mathrm{C} 15$ & $172.5(3)$ \\
\hline $\mathrm{N} 4-\mathrm{C} 14-\mathrm{C} 15-\mathrm{C} 16$ & $0.0(6)$ \\
\hline $\mathrm{C} 14-\mathrm{C} 15-\mathrm{C} 16-\mathrm{C} 17$ & $0.6(5)$ \\
\hline $\mathrm{C} 15-\mathrm{C} 16-\mathrm{C} 17-\mathrm{C} 25$ & $-0.8(5)$ \\
\hline
\end{tabular}




$\begin{array}{ll}\mathrm{N} 2-\mathrm{Cu} 1-\mathrm{N} 3-\mathrm{C} 12 \mathrm{~B} & -129.9(6) \\ \mathrm{O} 1-\mathrm{Cu} 1-\mathrm{N} 3-\mathrm{C} 11 \mathrm{~A} & -159.7(4) \\ \mathrm{N} 2-\mathrm{Cu} 1-\mathrm{N} 3-\mathrm{C} 11 \mathrm{~A} & 18.6(4) \\ \mathrm{O} 3-\mathrm{Cu} 2-\mathrm{N} 4-\mathrm{C} 14 & 96.8(6) \\ \mathrm{O} 2-\mathrm{Cu} 2-\mathrm{N} 4-\mathrm{C} 14 & 8.5(3) \\ \mathrm{N} 5-\mathrm{Cu} 2-\mathrm{N} 4-\mathrm{C} 14 & -178.4(3) \\ \mathrm{O} 4-\mathrm{Cu} 2-\mathrm{N} 4-\mathrm{C} 14 & -90.1(3) \\ \mathrm{O} 3-\mathrm{Cu} 2-\mathrm{N} 4-\mathrm{C} 25 & -90.1(6) \\ \mathrm{O} 2-\mathrm{Cu} 2-\mathrm{N} 4-\mathrm{C} 25 & -178.5(2) \\ \mathrm{N} 5-\mathrm{Cu} 2-\mathrm{N} 4-\mathrm{C} 25 & -5.3(2) \\ \mathrm{O} 4-\mathrm{Cu} 2-\mathrm{N} 4-\mathrm{C} 25 & 83.0(2) \\ \mathrm{O} 3-\mathrm{Cu} 2-\mathrm{N} 5-\mathrm{C} 23 & -15.8(4) \\ \mathrm{N} 4-\mathrm{Cu} 2-\mathrm{N} 5-\mathrm{C} 23 & 175.8(4) \\ \mathrm{O} 4-\mathrm{Cu} 2-\mathrm{N} 5-\mathrm{C} 23 & 80.5(4) \\ \mathrm{O} 3-\mathrm{Cu} 2-\mathrm{N} 5-\mathrm{C} 24 & 174.7(3) \\ \mathrm{N} 4-\mathrm{Cu} 2-\mathrm{N} 5-\mathrm{C} 24 & 6.4(3) \\ \mathrm{O} 4-\mathrm{Cu} 2-\mathrm{N} 5-\mathrm{C} 24 & -88.9(3) \\ \mathrm{Cu} 1-\mathrm{O} 1-\mathrm{C} 1-\mathrm{C} 6 & 175.6(3) \\ \mathrm{Cu} 1-\mathrm{O} 1-\mathrm{C} 1-\mathrm{C} 2 & -5.4(4) \\ \mathrm{C} 7-\mathrm{N} 1-\mathrm{C} 2-\mathrm{C} 3 & 3.4(6) \\ \mathrm{Cu} 1-\mathrm{N} 1-\mathrm{C} 2-\mathrm{C} 3 & -172.1(3) \\ \mathrm{C} 7-\mathrm{N} 1-\mathrm{C} 2-\mathrm{C} 1 & -177.8(3) \\ \mathrm{Cu} 1-\mathrm{N} 1-\mathrm{C} 2-\mathrm{C} 1 & 6.6(4) \\ \mathrm{O} 1-\mathrm{C} 1-\mathrm{C} 2-\mathrm{C} 3 & 178.2(3) \\ \mathrm{C} 6-\mathrm{C} 1-\mathrm{C} 2-\mathrm{C} 3 & -2.8(5) \\ \mathrm{O} 1-\mathrm{C} 1-\mathrm{C} 2-\mathrm{N} 1 & -0.7(4) \\ \mathrm{C} 6-\mathrm{C} 1-\mathrm{C} 2-\mathrm{N} 1 & 178.3(3) \\ \mathrm{N} 1-\mathrm{C} 2-\mathrm{C} 3-\mathrm{C} 4 & 179.7(3) \\ \mathrm{C} 1-\mathrm{C} 2-\mathrm{C} 3-\mathrm{C} 4 & 1.0(5) \\ \mathrm{C} 2-\mathrm{C} 3-\mathrm{C} 4-\mathrm{C} 5 & 1.0(6) \\ \mathrm{C} 3-\mathrm{C} 4-\mathrm{C} 5-\mathrm{C} 6 & -1.2(6) \\ \mathrm{C} 4-\mathrm{C} 5-\mathrm{C} 6-\mathrm{C} 1 & -0.6(6) \\ \mathrm{O} 1-\mathrm{C} 1-\mathrm{C} 6-\mathrm{C} 5 & -178.5(3) \\ \mathrm{C} 2-\mathrm{C} 1-\mathrm{C} 6-\mathrm{C} 5 & 2.6(5) \\ \mathrm{Cu} 2-\mathrm{O} 2-\mathrm{C} 7-\mathrm{N} 1 & 176.7(3) \\ \mathrm{Cu} 2-\mathrm{O} 2-\mathrm{C} 7-\mathrm{C} 8 & -5.4(4) \\ \mathrm{C} 2-\mathrm{N} 1-\mathrm{C} 7-\mathrm{O} 2 & 0.5(6) \\ \mathrm{Cu} 1-\mathrm{N} 1-\mathrm{C} 7-\mathrm{O} 2 & -177.5(3) \\ \mathrm{C} 2-\mathrm{N} 1-\mathrm{C} 7-\mathrm{C} 8 & \\ \mathrm{Cu} 1-\mathrm{N} 1-\mathrm{C} 7-\mathrm{C} 8 & (4) \\ & \end{array}$

$\begin{array}{ll}\mathrm{C} 15-\mathrm{C} 16-\mathrm{C} 17-\mathrm{C} 18 & -179.4(3) \\ \mathrm{C} 16-\mathrm{C} 17-\mathrm{C} 18-\mathrm{O} 5 & -6.4(6) \\ \mathrm{C} 25-\mathrm{C} 17-\mathrm{C} 18-\mathrm{O} 5 & 175.0(3) \\ \mathrm{C} 16-\mathrm{C} 17-\mathrm{C} 18-\mathrm{C} 19 & 174.4(3) \\ \mathrm{C} 25-\mathrm{C} 17-\mathrm{C} 18-\mathrm{C} 19 & -4.2(5) \\ \mathrm{O} 5-\mathrm{C} 18-\mathrm{C} 19-\mathrm{O} 6 & 5.9(6) \\ \mathrm{C} 17-\mathrm{C} 18-\mathrm{C} 19-\mathrm{O} 6 & -174.9(3) \\ \mathrm{O} 5-\mathrm{C} 18-\mathrm{C} 19-\mathrm{C} 20 & -176.5(3) \\ \mathrm{C} 17-\mathrm{C} 18-\mathrm{C} 19-\mathrm{C} 20 & 2.7(5) \\ \mathrm{O} 6-\mathrm{C} 19-\mathrm{C} 20-\mathrm{C} 21 & 0.5(6) \\ \mathrm{C} 18-\mathrm{C} 19-\mathrm{C} 20-\mathrm{C} 21 & -177.1(4) \\ \mathrm{O} 6-\mathrm{C} 19-\mathrm{C} 20-\mathrm{C} 24 & 178.4(4) \\ \mathrm{C} 18-\mathrm{C} 19-\mathrm{C} 20-\mathrm{C} 24 & 0.8(5) \\ \mathrm{C} 24-\mathrm{C} 20-\mathrm{C} 21-\mathrm{C} 22 & 1.6(6) \\ \mathrm{C} 19-\mathrm{C} 20-\mathrm{C} 21-\mathrm{C} 22 & 179.5(4) \\ \mathrm{C} 20-\mathrm{C} 21-\mathrm{C} 22-\mathrm{C} 23 & 0.6(7) \\ \mathrm{C} 24-\mathrm{N} 5-\mathrm{C} 23-\mathrm{C} 22 & 1.4(7) \\ \mathrm{Cu} 2-\mathrm{N} 5-\mathrm{C} 23-\mathrm{C} 22 & -167.4(4) \\ \mathrm{C} 21-\mathrm{C} 22-\mathrm{C} 23-\mathrm{N} 5 & -2.2(8) \\ \mathrm{C} 23-\mathrm{N} 5-\mathrm{C} 24-\mathrm{C} 20 & 0.9(6) \\ \mathrm{Cu} 2-\mathrm{N} 5-\mathrm{C} 24-\mathrm{C} 20 & 171.2(3) \\ \mathrm{C} 23-\mathrm{N} 5-\mathrm{C} 24-\mathrm{C} 25 & -176.6(4) \\ \mathrm{Cu} 2-\mathrm{N} 5-\mathrm{C} 24-\mathrm{C} 25 & -6.3(4) \\ \mathrm{C} 21-\mathrm{C} 20-\mathrm{C} 24-\mathrm{N} 5 & -2.4(6) \\ \mathrm{C} 19-\mathrm{C} 20-\mathrm{C} 24-\mathrm{N} 5 & 179.6(3) \\ \mathrm{C} 21-\mathrm{C} 20-\mathrm{C} 24-\mathrm{C} 25 & 175.0(4) \\ \mathrm{C} 19-\mathrm{C} 20-\mathrm{C} 24-\mathrm{C} 25 & -3.0(5) \\ \mathrm{C} 14-\mathrm{N} 4-\mathrm{C} 25-\mathrm{C} 17 & 0.0(5) \\ \mathrm{Cu} 2-\mathrm{N} 4-\mathrm{C} 25-\mathrm{C} 17 & -173.8(3) \\ \mathrm{C} 14-\mathrm{N} 4-\mathrm{C} 25-\mathrm{C} 24 & 177.2(3) \\ \mathrm{Cu} 2-\mathrm{N} 4-\mathrm{C} 25-\mathrm{C} 24 & 3.4(4) \\ \mathrm{C} 16-\mathrm{C} 17-\mathrm{C} 25-\mathrm{N} 4 & 0.5(5) \\ \mathrm{C} 18-\mathrm{C} 17-\mathrm{C} 25-\mathrm{N} 4 & 179.1(3) \\ \mathrm{C} 16-\mathrm{C} 17-\mathrm{C} 25-\mathrm{C} 24 & -176.4(3) \\ \mathrm{C} 18-\mathrm{C} 17-\mathrm{C} 25-\mathrm{C} 24 & 2.2(5) \\ \mathrm{N} 5-\mathrm{C} 24-\mathrm{C} 25-\mathrm{N} 4 & 2.0(5) \\ \mathrm{C} 20-\mathrm{C} 24-\mathrm{C} 25-\mathrm{N} 4 & -175.6(3) \\ \mathrm{N} 5-\mathrm{C} 24-\mathrm{C} 25-\mathrm{C} 17 & 179.2(3) \\ \mathrm{C} 20-\mathrm{C} 24-\mathrm{C} 25-\mathrm{C} 17 & 1.6(5) \\ & \end{array}$

Hydrogen-bond geometry $\left(\AA,{ }^{\circ}\right)$

\begin{tabular}{lllll}
\hline$D-\mathrm{H} \cdots A$ & $D-\mathrm{H}$ & $\mathrm{H} \cdots A$ & $D \cdots A$ & $D-\mathrm{H} \cdots A$ \\
\hline $\mathrm{O} 4-\mathrm{H} 4 A \cdots \mathrm{O} 1^{\mathrm{i}}$ & 0.84 & 1.89 & $2.666(3)$ & 153 \\
$\mathrm{O} 4-\mathrm{H} 4 B \cdots \mathrm{O} 10 B$ & 0.91 & 1.93 & $2.740(8)$ & 146 \\
$\mathrm{O} 4-\mathrm{H} 4 B \cdots \mathrm{O} 11 A$ & 0.91 & 2.10 & $3.009(7)$ & 171 \\
$\mathrm{O} 7 A-\mathrm{H} 7 A \cdots \mathrm{O} 3$ & 0.86 & 2.51 & $3.27(2)$ & 147
\end{tabular}


supporting information

$\begin{array}{lllll}\mathrm{O} 7 B-\mathrm{H} 7 D \cdots \mathrm{O} 5^{\mathrm{ii}} & 0.86 & 2.51 & 3.305(18) & 153 \\ \mathrm{C} 3-\mathrm{H} 3 \cdots \mathrm{O} 8^{\mathrm{iii}} & 0.93 & 2.52 & 3.193(5) & 130 \\ \mathrm{C} 15-\mathrm{H} 15 \cdots \mathrm{O} 9 A^{\mathrm{iii}} & 0.93 & 2.55 & 3.335(7) & 142 \\ \mathrm{C} 15-\mathrm{H} 15 \cdots \mathrm{O} 11 B^{\mathrm{iii}} & 0.93 & 2.49 & 3.384(10) & 162 \\ \mathrm{C} 16-\mathrm{H} 16 \cdots 6^{\mathrm{iii}} & 0.93 & 2.54 & 3.195(5) & 128 \\ \mathrm{C} 10 A-\mathrm{H} 10 A \cdots \mathrm{O} 6^{\mathrm{ii}} & 0.97 & 2.44 & 3.202(9) & 135 \\ \mathrm{C} 11 A-\mathrm{H} 11 A \cdots \mathrm{O} 4^{\mathrm{iv}} & 0.97 & 2.54 & 3.409(7) & 150 \\ \mathrm{C} 13 A-\mathrm{H} 13 B \cdots \mathrm{O} 4^{\mathrm{i}} & 0.96 & 2.49 & 3.382(16) & 154 \\ \mathrm{C} 21-\mathrm{H} 21 \cdots \mathrm{O} 10 A^{\mathrm{v}} & 0.93 & 2.53 & 3.277(8) & 138 \\ \mathrm{C} 23-\mathrm{H} 23 \cdots \mathrm{O} 7 A & 0.93 & 2.30 & 3.154(19) & 152 \\ \mathrm{C} 23-\mathrm{H} 23 \cdots \mathrm{O} 7 B & 0.93 & 2.49 & 3.31(2) & 147\end{array}$

Symmetry codes: (i) $-x+1,-y+1,-z$; (ii) $-x, y+1 / 2,-z+1 / 2$; (iii) $x,-y+1 / 2, z-1 / 2$; (iv) $-x+1, y+1 / 2,-z+1 / 2$; (v) $x,-y+1 / 2, z+1 / 2$. 